MATHEMATICS OF COMPUTATION

Volume 76, Number 258, April 2007, Pages 903-929

S 0025-5718(06)01949-1

Article electronically published on December 28, 2006

\title{
ON INTERPOLATORY DIVERGENCE-FREE WAVELETS
}

\author{
KAI BITTNER AND KARSTEN URBAN
}

\begin{abstract}
We construct interpolating divergence-free multiwavelets based on cubic Hermite splines. We give characterizations of the relevant function spaces and indicate their use for analyzing experimental data of incompressible flow fields. We also show that the standard interpolatory wavelets, based on the Deslauriers-Dubuc interpolatory scheme or on interpolatory splines, cannot be used to construct compactly supported divergence-free interpolatory wavelets.
\end{abstract}

\section{INTRODUCTION}

Wavelets are by now a widely accepted tool in signal and image processing as well as in numerical simulation. Divergence-free vector fields have obvious possible applications for the analysis and numerical simulation of incompressible flows typically modeled by the Stokes system or by the incompressible Navier-Stokes equations. This has lead to the construction of divergence-free wavelets. The first construction was introduced in [3] leading to an orthonormal wavelet basis for the space of divergence-free vector fields on $\mathbb{R}^{n}$. These functions were used in [17] to show an existence result for the instationary, incompressible Navier-Stokes equations on $\mathbb{R}^{n}$. For numerical purposes, in particular for bounded domains, these wavelet bases are not appropriate since they are globally supported. In fact, it was shown in 27] that no orthonormal, compactly supported divergence-free wavelet basis exists.

In 26, 32 compactly supported biorthogonal wavelets have been constructed and these functions have been used for the analysis of the Navier-Stokes equations 28 , the numerical simulation of the Stokes equations on rectangular domains 34 and also for the analysis of experimental data of incompressible turbulent flows [1]. There are also generalizations to multiwavelets [24, 25].

However, the application of divergence-free wavelets in numerical simulations is so far restricted to rather academic simple domains such as rectangles. This is mainly due to the necessity of a rather technically complicated construction of biorthogonal divergence-free wavelet bases on general domain geometries. This is one reason for considering collocation methods; see e.g. 222, 23, 35, 37. In this case, interpolatory wavelets are required, which can easily be adapted to complicated domains. However, to our knowledge, there are no divergence-free interpolatory wavelets available, which is the reason why, e.g. in [22], an additional projection method has to be performed.

Received by the editor March 18, 2005 and, in revised form, March 13, 2006.

2000 Mathematics Subject Classification. Primary 42C40, 35Q30, 41A15.

Key words and phrases. Interpolatory wavelets, divergence-free vector fields.

We are grateful to Nicolas Kevlahan for bringing our attention to the topic of this paper.

(c)2006 American Mathematical Society Reverts to public domain 28 years from publication 
Another motivation for our investigation comes from applications of wavelets in data analysis. Since wavelets are a widely accepted tool for signal and image processing, denoising and compression, it is a natural idea to use wavelet methods also for the analysis of incompressible flow data. Of course, for incompressible flows, divergence-free wavelets would be appropriate. However, since typically the data in terms of point values is approximated by scaling function coefficients this does not only invent an approximation error, it also adds artificial compressibility (as in [1). This could be avoided if point values and expansion coefficients coincide, which is the case for interpolatory functions.

The remainder of this paper is organized as follows. In Section 2 we collect some preliminaries, notation, and basics on interpolatory wavelets and we review the main construction idea for divergence-free wavelets. It is a natural idea to consider the most frequently used interpolatory wavelet bases as a starting point for the construction of interpolatory divergence-free wavelets. We show in Section 2.2 .2 that this approach does not work. Thus, we consider a multiwavelet construction based on cubic Hermite splines in Section 3. We show in Section 4 how our construction can be used within the lifting scheme. Further generalization to arbitrary grids and domains as well as the numerical applications are devoted to a forthcoming paper.

\section{Preliminaries}

In this section, we collect some preliminary facts that are needed in the remainder of the paper. Let us first set our notation. Let $\Omega \subseteq \mathbb{R}^{n}$ be a domain, $L_{p}(\Omega)$ the standard Lebesgue space, and

$$
\boldsymbol{L}_{p}(\Omega):=L_{p}(\Omega)^{n}:=\left\{\boldsymbol{v}=\left(v_{1}, \ldots, v_{n}\right)^{T}: v_{i} \in L_{p}(\Omega), i=1, \ldots, n\right\}
$$

the corresponding space of vector fields. We will always denote vector fields by boldface characters and real-valued functions in the standard font. For example, $\boldsymbol{H}^{k}(\Omega)$ is the space of $n$-dimensional vector fields whose components are functions in $H^{k}(\Omega)$.

The spaces of vector-fields induced by the divergence operator are denoted by

$$
\begin{aligned}
\boldsymbol{H}(\operatorname{div} ; \Omega) & :=\left\{\boldsymbol{v} \in \boldsymbol{L}_{2}(\Omega): \operatorname{div} \boldsymbol{v} \in L_{2}(\Omega)\right\}, \\
\boldsymbol{V}(\operatorname{div} ; \Omega) & :=\{\boldsymbol{v} \in \boldsymbol{H}(\operatorname{div} ; \Omega): \operatorname{div} \boldsymbol{v}=0\} .
\end{aligned}
$$

Since we consider interpolatory functions, we will also need the spaces $\mathbf{C}^{k}(\Omega)$ and

$$
\begin{aligned}
\boldsymbol{C}(\operatorname{div} ; \Omega) & :=\{\boldsymbol{v} \in \boldsymbol{C}(\Omega): \operatorname{div} \boldsymbol{v} \in C(\Omega)\}, \\
\boldsymbol{N}(\operatorname{div} ; \Omega) & :=\{\boldsymbol{v} \in \boldsymbol{C}(\operatorname{div} ; \Omega): \operatorname{div} \boldsymbol{v}=0\} .
\end{aligned}
$$

We will frequently use the notation $A \lesssim B$ to abbreviate that $A$ is bounded by a constant multiple of $B . A \gtrsim B$ is defined as $B \lesssim A$ and $A \sim B$ means $A \lesssim B$ and $A \gtrsim B$.

2.1. How to construct divergence-free wavelets. A standard tool for the construction of wavelet bases for $L_{2}(\mathbb{R})$, i.e., on the whole real line, is a multiresolution analysis (MRA), which is a nested sequence of spaces $V_{j} \subset V_{j+1} \subset L_{2}(\mathbb{R}), j \in \mathbb{Z}$, whose union is dense in $L_{2}(\mathbb{R})$, while the intersection contains only the zero function. Moreover, $V_{0}$ is shift-invariant and $V_{j}$ arises from $V_{0}$ by scaling in the sense that $v \in V_{0}$ if and only if $v\left(2^{j} \cdot\right) \in V_{j}$. Finally, these spaces are generated by a scaling function $\xi$ in the sense that

$$
V_{j}=\operatorname{clos}_{L_{2}} \operatorname{span}\left\{\xi_{j, k}: k \in \mathbb{Z}\right\}
$$


where $\xi_{j, k}(x):=2^{j / 2} \xi\left(2^{j} x-k\right), x \in \mathbb{R}$, is a scaled and shifted version of $\xi$. From $V_{0} \subset V_{1}$ it follows immediately that $\xi$ is refinable, i.e.,

$$
\xi(x)=\sum_{k \in \mathbb{Z}} a_{k} \xi(2 x-k), \quad x \in \mathbb{R} .
$$

The translates of $\xi$ should be orthonormal or at least stable, in the sense that

$$
\left\|\sum_{k \in \mathbb{Z}} c_{k} \xi(2 \cdot-k)\right\|_{L_{2}(\mathbb{R})} \sim\|\boldsymbol{c}\|_{\ell_{2}(\mathbb{Z})}, \quad \boldsymbol{c}=\left(c_{k}\right)_{k \in \mathbb{Z}} \in \ell_{2}(\mathbb{Z}) .
$$

A MRA can also be constructed on bounded domains $\Omega \subset \mathbb{R}^{n}$, involving more technicalities and renouncing shift-invariance, of course; see, e.g. 6, 7, 10, 12, 13, 14.

Once a MRA (or two in the case of biorthogonal wavelets) is known, there are several general techniques to construct a corresponding wavelet basis.

Thus, it would be an immediate idea to construct a divergence-free wavelet basis from a MRA in $\boldsymbol{V}(\operatorname{div} ; \Omega)$. However, as shown in [26, there is no stable divergencefree compactly supported scaling function generating a MRA. Instead, one uses an appropriate MRA $\left\{\boldsymbol{V}_{j}: j \in \mathbb{Z}\right\}$ in $\boldsymbol{H}(\operatorname{div} ; \Omega)$ and then splits the detail spaces

$$
\boldsymbol{W}_{j}:=\boldsymbol{V}_{j+1} \ominus \boldsymbol{V}_{j}=\boldsymbol{W}_{j}^{\nabla} \oplus \boldsymbol{W}_{j}^{\Delta}
$$

into the divergence-free part $\boldsymbol{W}_{j}^{\nabla}=\boldsymbol{W}_{j} \cap \boldsymbol{V}(\operatorname{div} ; \Omega)$ and a stable complement $\boldsymbol{W}_{j}^{\Delta}$. Then, one needs to construct a wavelet basis $\left\{\boldsymbol{\psi}_{j, k}^{\nabla}: k\right\}$ for $\boldsymbol{W}_{j}^{\nabla}$ and the collection over all levels $j$ gives the desired basis of divergence-free wavelets.

Biorthogonal wavelets, differentiation, and integration. Next, we need to describe what an "appropriate" MRA in $\boldsymbol{H}(\operatorname{div} ; \Omega)$ is. In order to avoid unnecessary technicalities we restrict ourselves to the case $\Omega=\mathbb{R}^{n}$ and refer to [33, 34] for the construction on general bounded domains. Keeping in mind that any divergencefree wavelet $\boldsymbol{\psi}_{j, k}^{\nabla}$ is a linear combination of the basis functions of $\boldsymbol{V}_{j+1}$, the spaces

$$
V_{j}^{\operatorname{div}}:=\operatorname{div}\left(\boldsymbol{V}_{j}\right) \subset L_{2}\left(\mathbb{R}^{n}\right)
$$

play an important role in the construction. Recall that $\boldsymbol{V}_{j}$ is a vector-valued multiresolution space, i.e., the spaces are of the form

$$
\boldsymbol{V}_{j}=V_{j}^{1} \times \cdots \times V_{j}^{n},
$$

where each $V_{j}^{i}$ is a real-valued multiresolution space. Hence, a natural condition is to require that

$$
\frac{\partial}{\partial x_{i}} V_{j}^{i}=V_{j}^{\text {div }}, \quad i=1, \ldots, n,
$$

where $V_{j}^{\text {div }}$ is a multiresolution space, too.

In turn, if we use a standard tensor product construction to obtain a MRA on $\mathbb{R}^{n}$ from $n$ MRA on $\mathbb{R},(2.8)$ reduces to a relation of the form

$$
\frac{d}{d x} V_{j}^{+}=V_{j}^{-}
$$

for two multiresolution spaces $V_{j}^{+}, V_{j}^{-}$of functions $f_{j}: \mathbb{R} \rightarrow \mathbb{R}$. Indeed, one can choose $V_{j}^{\text {div }}:=V_{j}^{-} \otimes \ldots \otimes V_{j}^{-}$and $V_{j}^{i}:=V_{j}^{-} \otimes \ldots \otimes V_{j}^{-} \otimes V_{j}^{+} \otimes V_{j}^{-} \otimes \ldots \otimes V_{j}^{-}$ with $V_{j}^{+}$as the $i$-th factor in the above tensor product. 
For the corresponding compactly supported scaling functions this implies the relation

$$
\frac{d}{d x} \xi^{+}(x)=\sum_{k=-d}^{d} \alpha_{k} \xi^{-}(x-k)
$$

for some finite $d \in \mathbb{N}$ and coefficients $\alpha_{k} \in \mathbb{R}$. Note that $d<\infty$ because $\xi^{ \pm}$ are assumed to be compactly supported and stable. We will use $\xi$ to indicate a univariate scaling function while $\varphi$ will always denote a multivariate function.

It is proven in [26] that for a biorthogonal pair $\xi^{+} \in H^{1}(\mathbb{R}), \tilde{\xi}^{+} \in L_{2}(\mathbb{R})$ of compactly supported scaling functions there always exists another biorthogonal pair $\xi^{-} \in L_{2}(\mathbb{R}), \tilde{\xi}^{-} \in H^{1}(\mathbb{R})$ of compactly supported scaling functions such that

$$
\frac{d}{d x} \xi^{+}(x)=\xi^{-}(x)-\xi^{-}(x-1), \quad \frac{d}{d x} \tilde{\xi}^{-}(x)=\tilde{\xi}^{+}(x+1)-\tilde{\xi}^{+}(x) .
$$

Note that $\tilde{\xi}^{ \pm}$denotes the dual of $\xi^{ \pm}$in order to avoid the more cumbersome notation $\widetilde{\xi^{ \pm}}$. Moreover, the corresponding biorthogonal wavelets satisfy the relation

$$
\frac{d}{d x} \eta^{+}(x)=4 \eta^{-}(x), \quad \frac{d}{d x} \tilde{\eta}^{-}(x)=-\frac{1}{4} \tilde{\eta}^{+}(x) .
$$

From the above remarks, it should be clear that the latter equations are the heart of the construction of divergence-free wavelets. We will come back to this point later in Section 2.2 .2

Now, we define for any subset of indices $I \subseteq\{1, \ldots, n\}$,

$$
V_{j}^{I}:=\bigotimes_{\nu=1}^{n} V_{j}^{(I, \nu)}, \text { where } V_{j}^{(I, \nu)}:= \begin{cases}V_{j}^{+}, & \text {if } \nu \in I, \\ V_{j}^{-}, & \text {if } \nu \notin I .\end{cases}
$$

In particular, we set, as already mentioned above, $V_{j}^{\text {div }}:=V_{j}^{\emptyset}=V_{j}^{-} \otimes \cdots \otimes V_{j}^{-}$ and $V_{j}^{i}:=V_{j}^{\{i\}}$. The corresponding scaling functions are

$$
\varphi^{I}\left(x_{1}, \ldots, x_{n}\right):=\prod_{\nu=1}^{n} \xi_{\nu}^{I}\left(x_{\nu}\right), \quad \text { where } \xi_{\nu}^{I}:= \begin{cases}\xi^{+}, & \text {if } \nu \in I, \\ \xi^{-}, & \text {if } \nu \notin I .\end{cases}
$$

Obviously, the vector fields

$$
\boldsymbol{\varphi}_{i}:=\varphi^{\{i\}} \boldsymbol{\delta}_{i}
$$

are scaling functions in $\boldsymbol{H}\left(\operatorname{div} ; \mathbb{R}^{n}\right)$, where $\boldsymbol{\delta}_{i}:=\left(\delta_{1, i}, \ldots, \delta_{n, i}\right)^{T}$ denotes the $i$-th canonical vector in $\mathbb{R}^{n}$. In fact,

$$
\boldsymbol{V}_{j}:=\operatorname{clos} \operatorname{span}\left\{\boldsymbol{\varphi}_{i ; j, k}, i=1, \ldots, n, k \in \mathbb{Z}^{n}\right\}
$$

is the desired MRA, where

$$
\boldsymbol{\varphi}_{i ; j, k}(x):=2^{(n j) / 2} \boldsymbol{\varphi}_{i}\left(2^{j} x-k\right) \quad x \in \mathbb{R}^{n}, j \in \mathbb{Z}, k \in \mathbb{Z}^{n} .
$$

Divergence-free wavelets. Now, we consider the corresponding wavelets. Let $\eta^{+}$, $\eta^{-}$be (orthogonal or biorthogonal) wavelets according to $\xi^{+}$and $\xi^{-}$, respectively. Then, the wavelets generated by $\varphi^{I}$ are defined for

$$
E_{n}:=\{0,1\}^{n} \text { and } E_{n}^{*}:=E_{n} \backslash\{0\}
$$

as

$$
\psi_{e}^{I}:=\bigotimes_{\nu=1}^{n} \vartheta_{e_{\nu}, \nu}^{I}, \quad \text { where } \quad \vartheta_{\ell, \nu}^{I}:= \begin{cases}\xi_{\nu}^{I}, & \text { if } \ell=0 \\ \eta_{\nu}^{I}, & \text { if } \ell=1\end{cases}
$$


with $\eta_{\nu}^{I}$ defined analogously to $\xi_{\nu}^{I}$ in (2.13). The vector-valued wavelets in $\boldsymbol{H}\left(\operatorname{div} ; \mathbb{R}^{n}\right)$ are scaled translates $\boldsymbol{\psi}_{e, i ; j, k}$ of the mother wavelets

$$
\boldsymbol{\psi}_{e, i}:=\psi_{e}^{\{i\}} \boldsymbol{\delta}_{i}, \quad e \in E_{n}^{*}
$$

Note that for any $e \in E_{n}^{*}$, there exists an index

$$
i_{e} \in\{1, \ldots, n\} \text { such that } e_{i_{e}}=1 \text {, }
$$

i.e., at least one factor in the tensor product is a univariate wavelet. This index plays a crucial role in the definition of divergence-free wavelets. In fact, the additional constraint div $\boldsymbol{u}=0$ fixes one degree of freedom. This means that we have $\left|E_{n}^{*}\right|(n-$ 1) $=\left(2^{n}-1\right)(n-1)$ wavelets instead of $n\left|E_{n}^{*}\right|$ (which is the number of wavelets in $\boldsymbol{L}_{2}(\Omega)$ ). Then, we define divergence-free wavelets for $e \in E_{n}^{*}$ and $i \neq i_{e}$ componentwise by

$$
\boldsymbol{\psi}_{e, i: \nu}^{\nabla}:= \begin{cases}\psi_{e}^{\{i\}}, & \text { if } \nu=i ; \\ -\frac{1}{4} \frac{\partial}{\partial x_{i}} \psi_{e}^{\left\{i, i_{e}\right\}}, & \text { if } \nu=i_{e} ; \\ 0 & \text { otherwise }\end{cases}
$$

We always denote by $\boldsymbol{f}_{: i}$ the $i$-th component of a vector field $\boldsymbol{f}$. Note that \#\{(e,i): $\left.e \in E^{*}, i \neq i_{e}\right\}=\left|E^{*}\right|(n-1)=\left(2^{n}-1\right)(n-1)$, so that we have in fact the correct number of divergence-free wavelets. It is easily seen that these functions are divergence-free, i.e.,

$$
\begin{aligned}
\operatorname{div} \boldsymbol{\psi}_{e, i}^{\nabla} & =\frac{\partial}{\partial x_{i}} \boldsymbol{\psi}_{e, i: i}^{\nabla}+\frac{\partial}{\partial x_{i_{e}}} \boldsymbol{\psi}_{e, i: i_{e}}^{\nabla}=\frac{\partial}{\partial x_{i}} \psi_{e}^{\{i\}}-\frac{1}{4} \frac{\partial}{\partial x_{i_{e}}} \frac{\partial}{\partial x_{i}} \psi_{e}^{\left\{i, i_{e}\right\}} \\
& =\frac{1}{4} \frac{\partial}{\partial x_{i}} \frac{\partial}{\partial x_{i_{e}}} \psi_{e}^{\left\{i, i_{e}\right\}}-\frac{1}{4} \frac{\partial}{\partial x_{i_{e}}} \frac{\partial}{\partial x_{i}} \psi_{e}^{\left\{i, i_{e}\right\}}=0 .
\end{aligned}
$$

Note, that the index $i_{e}$ is chosen so that we can apply equality (2.11). Moreover, it was shown in [26, 32, that the system

$$
\boldsymbol{\Psi}^{\nabla}:=\left\{\boldsymbol{\psi}_{e, i}^{\nabla}: e \in E^{*}, i \neq i_{e}\right\}
$$

generates a Riesz basis for the space $\boldsymbol{V}\left(\operatorname{div} ; \mathbb{R}^{n}\right)$ of all divergence-free vector fields on $\mathbb{R}^{n}$. Generalizations to more general domains can be found in [33, 34].

As already mentioned, the above system is a biorthogonal one. Let us denote by the accent " " the dual system corresponding to all functions involved in the construction. Then, the dual wavelets according to $\boldsymbol{\psi}_{e, i}^{\nabla}$ are defined by

$$
\tilde{\boldsymbol{\psi}}_{e, i}^{\nabla}:=\tilde{\psi}_{e}^{\{i\}} \boldsymbol{\delta}_{i}, \quad e \in E^{*}, i \neq i_{e} .
$$

Note that these functions are not divergence-free which is by no means a contradiction. It comes from the splitting of the complement spaces in (2.7).

2.2. Interpolatory wavelets. As above, the starting point for the construction of interpolatory wavelets is a MRA generated by an interpolatory scaling function $\xi$, i.e., $\xi(k)=\delta_{0, k}$ for all $k \in \mathbb{Z}$. Thus, any function $f_{j} \in V_{j}$ has an expansion of the form

$$
f_{j}(x)=\sum_{k \in \mathbb{Z}} c_{j, k} \xi\left(2^{j} x-k\right), \quad c_{j, k}=f\left(2^{-j} k\right),
$$

i.e., the expansion coefficients are given in terms of point values (samples) of $f$ rather than by a scalar product $\left(f, \tilde{\xi}_{j, k}\right)_{0, \Omega}$. 
It is readily seen that

$$
\eta(x):=2 \xi(2 x-1)
$$

is a possible choice for such a wavelet with interpolatory properties, [16. This is also the reason, why in several papers the term "interpolatory wavelet" is used for the scaling function $\xi$. From $(2.19)$ it is obvious that interpolatory wavelets do not have vanishing moments. On the other hand, one still has fast algorithms for decomposition and reconstruction of a signal (Fast Wavelet Transform). Finally, one cannot hope to obtain a Riesz basis for $L_{2}$. However, in [16, norm equivalences and characterizations for Besov spaces embedded in $C(\mathbb{R})$ are proven (the range of Besov spaces depends of course on the regularity and polynomial exactness of $\xi$ ); see also Theorem 2.1 below.

2.2.1. Characterization of function spaces. The following general characterization result is well-known. We will derive similar results for interpolatory divergence-free wavelets. Here $B_{q}^{s}\left(L_{p}(\mathbb{R})\right)$ denotes the standard Besov space.

Theorem 2.1 ([16, Thm. 2.7]). Let $\xi \in C^{r}(\mathbb{R})$ be a compactly supported, refinable, interpolatory scaling function which is exact of order $d$, i.e.,

$$
x^{m}=\sum_{k \in \mathbb{Z}} \alpha_{m k} \xi(x-k), \quad 0 \leq m \leq d, x \in \mathbb{R},
$$

with appropriate coefficients $\alpha_{m k} \in \mathbb{R}$, where the convergence of the sum is to be understood locally. Then we have the following characterization for the interpolatory wavelets $\eta=\xi(2 \cdot-1)$ for $\frac{1}{p}<s<\min \{r, d\}, 0<p, q \leq \infty$,

$$
\left\|\sum_{j, k} d_{j, k} \eta_{j, k}\right\|_{B_{q}^{s}\left(L_{p}(\mathbb{R})\right)} \sim\left(\sum_{j}\left(2^{j\left(s+\frac{1}{2}-\frac{1}{p}\right)} \sum_{k}\left|d_{j, k}\right|^{p}\right)^{q}\right)^{1 / q} .
$$

Note that the above result also holds for interpolatory spline functions (which are not compactly supported, but piecewise polynomials). In this case, one obviously has $r=d-1$.

2.2.2. Differentiation and integration. Let us now investigate the differentiation process as described in (2.9) for the particular case of interpolatory scaling functions.

Proposition 2.2. Let $\xi^{+} \in C^{1}(\mathbb{R})$ be a compactly supported, interpolatory scaling function with refinement coefficients $a_{k}^{+}$in (2.6). Then, the coefficients

$$
a_{k}^{-}:=\frac{1}{2}\left(a_{k}+a_{k-1}\right)
$$

define a refinable function $\xi^{-}$satisfying

$$
\frac{d}{d x} \xi^{+}(x)=\xi^{-}(x)-\xi^{-}(x-1) .
$$

We skip the proof since it is an immediate consequence of [26, Prop. 4]. In order to use this differentiation process as the starting point for a construction of divergence-free interpolatory wavelets, we investigate if (2.21) gives rise to an interpolatory scaling function $\xi^{-}$. In the following we comment on three prominent examples of interpolatory scaling functions. 
1. A sufficient but not necessary condition for $\xi^{-}$to be interpolatory is $a_{2 k}=\delta_{k}$, $k \in \mathbb{Z}$; see e.g. [29]. Assuming that this holds for $a_{k}^{+}$, we obtain by (2.21)

$$
a_{2 k}^{-}=\frac{1}{2}\left(\delta_{k}+a_{2 k-1}^{+}\right)
$$

which only coincides with $\delta_{k}$ if $a_{2 k-1}^{+}=\delta_{k}$. This, however, is only true for the scaling function corresponding to the orthonormal Haar wavelet.

2. One popular choice for the construction of wavelets are spline functions. Choosing the space of cardinal splines of even order $d>0$ as $V_{0}^{+}$, we can choose the corresponding fundamental spline as interpolatory scaling function $\xi^{+}$. However, then $V_{0}^{-}$is the space of splines of odd order, where the interpolation problem at the integer knots does not have a unique solution. Thus, we cannot have an interpolatory scaling function $\xi^{-}$; i.e., splines are not suited for the construction of interpolatory divergence-free wavelets. Moreover, $\xi^{+}$is only compactly supported for $d=2$ (i.e., the hat function).

3. The Deslauriers-Dubuc interpolatory scaling functions $\xi_{N}$ are defined as auto-correlation of Daubechies' orthonormal scaling functions $\theta_{N}$,

$$
\xi_{N}(x)=\left(\theta_{N} * \theta_{N}\right)(x):=\int_{\mathbb{R}} \theta_{N}(t) \theta_{N}(t-x) d t, \quad x \in \mathbb{R} .
$$

If we use $\xi^{+}=\xi_{N}$ (and $\theta^{+}:=\theta_{N}$ ), the arising function $\xi^{-}$is not interpolatory. In fact, we have

$$
\frac{d}{d x} \xi^{+}=\left(\frac{d}{d x} \theta^{+}\right) * \theta^{+}=\left(\theta^{-} * \theta^{+}\right)(x)-\left(\theta^{-} * \theta^{+}\right)(x-1),
$$

i.e., $\xi^{-}=\theta^{-} * \theta^{+}$. It is known that $\theta^{-}$is biorthogonal (and not interpolatory) and thus $\xi^{-}$is not interpolating at the integers.

\section{HeRmite INTERPolatory SPLiNES AND MULTiWAVELETS}

As we have seen in Section 2.2.2 above, it does not seem to be a good idea to try to construct interpolatory divergence-free wavelets starting from a scaling system generated by only one function $\xi^{+}$. Thus, we now aim to construct two families of functions $\Xi^{+}=\left\{\xi_{1}^{+}, \ldots, \xi_{m}^{+}\right\}$and $\Xi^{-}=\left\{\xi_{1}^{-}, \ldots, \xi_{m}^{-}\right\}$such that

- the derivatives of $\Xi^{+}$are spanned in terms of $\Xi^{-}$;

- both systems are interpolatory with respect to the integers.

This can in fact be achieved by Hermite interpolatory spline functions and leads us to multiwavelets.

Definition 3.1. The space of cardinal splines of order $d, d \geq 0$, with knots of multiplicity $i$ (splines with deficiency $i-1$ ) is defined by

$$
S_{d, i}:=\left\{f \in C^{d-i-1}(\mathbb{R}):\left.f\right|_{[k, k+1)} \in \Pi_{d-1}, k \in \mathbb{Z}\right\} .
$$

A stable basis of $S_{d, i}$ is induced by the B-splines

$$
B_{k}^{d, i}(x)=\left(t_{i, k+d}-t_{i, k}\right)\left[t_{i, k}, \ldots, t_{i, k+d}\right](\cdot-x)_{+}^{d-1}, \quad k \in \mathbb{Z},
$$

with knots $t_{i, k}:=\left\lfloor\frac{k}{i}\right\rfloor$ (see e.g. [30 $\rfloor$ ). In fact, the B-spline basis can be represented by integer translates of the $i$ successive B-splines $B_{k}^{d, i}, \ldots, B_{k+i-1}^{d, i}$. Splines with knots of multiplicity $i$ can be used to interpolate not only function values but also the values of derivatives up to order $i-1$ if the splines are sufficiently smooth, i.e., if $d \geq 2 i$. 

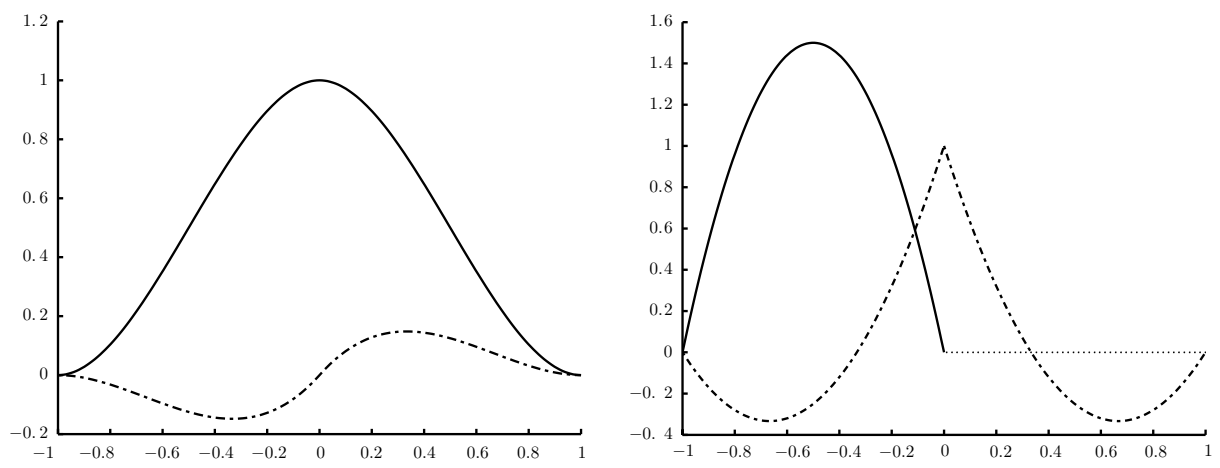

Figure 1. Original (left) and differentiated system of Hermite interpolatory splines.

3.1. Interpolatory Hermite splines and differentiation. In the sequel we consider cubic Hermite splines defined as follows:

$$
\begin{aligned}
& \xi_{1}^{+}(x):= \begin{cases}1-3 x^{2}-2 x^{3}, & \text { if } x \in[-1,0] ; \\
1-3 x^{2}+2 x^{3}, & \text { if } x \in[0,1] ; \\
0 & \text { otherwise, }\end{cases} \\
& \xi_{2}^{+}(x):= \begin{cases}x+2 x^{2}+x^{3}, & \text { if } x \in[-1,0] ; \\
x-2 x^{2}+x^{3}, & \text { if } x \in[0,1] ; \\
0 & \text { otherwise, }\end{cases}
\end{aligned}
$$

as well as the quadratic Hermite splines

$$
\begin{gathered}
\xi_{1}^{-}(x):= \begin{cases}-6 x-6 x^{2}, & \text { if } x \in[-1,0] ; \\
0 & \text { otherwise }\end{cases} \\
\xi_{2}^{-}(x):= \begin{cases}1+4 x+3 x^{2}, & \text { if } x \in[-1,0] ; \\
1-4 x+3 x^{2}, & \text { if } x \in[0,1] ; \\
0 & \text { otherwise. }\end{cases}
\end{gathered}
$$

The corresponding functions are displayed in Figure 1. These functions have been investigated in several papers. Biorthogonal multiwavelets on the interval $[0,1]$ generated by $\xi_{1}^{+}, \xi_{2}^{+}$have been introduced in [11. It is well known that the integer translates of $\xi_{1}^{+}, \xi_{2}^{+}$generate the space of $C^{1}$-continuous piecewise cubic functions on $\mathbb{R}$ which interpolate function values and first derivatives at the integers. Based on these functions, biorthogonal divergence-free multiwavelets (also on rectangular domains) have been constructed in [24, 25]. There, the system $\xi_{1}^{+}, \xi_{2}^{+}$plays a role as part of a whole family of multiwavelets that can be linked by differentiation and integration similar to (2.10).

The above functions are fundamental interpolants in the sense that

$$
\frac{d^{\nu}}{d x^{\nu}} \xi_{m}^{+}(k)=\delta_{k 0} \delta_{m-1, \nu}, \quad k \in \mathbb{Z}, \nu \in\{0,1\}, m \in\{1,2\},
$$

and

$$
\xi_{m}^{-}(k)=\delta_{k 0} \delta_{m 2}, \quad \int_{k-1}^{k} \xi_{m}^{-}(x) d x=\delta_{k 0} \delta_{m 1}, \quad k \in \mathbb{Z}, m \in\{1,2\}
$$


Furthermore, it is easily seen that we have the following relation for the derivatives

$$
\frac{d}{d x} \xi_{1}^{+}(x)=\xi_{1}^{-}(x)-\xi_{1}^{-}(x-1), \quad \frac{d}{d x} \xi_{2}^{+}(x)=\xi_{2}^{-}(x),
$$

which will turn out to be crucial later.

It is well known, that $\left\{\xi_{1}^{+}(\cdot-k), \xi_{2}^{+}(\cdot-k): k \in \mathbb{Z}\right\}$ is a stable basis for $S_{4,2}$. Indeed, this property follows immediately from $\xi_{1}^{+}=B_{-1}^{4,2}+B_{-2}^{4,2}$ and $\xi_{2}^{+}=\frac{1}{3}\left(B_{-1}^{4,2}-\right.$ $\left.B_{-2}^{4,2}\right)$. Furthermore, from $\xi_{1}^{-}(x)=3 B_{-2}^{3,2}$ and $\xi_{2}^{-}=3\left(B_{-1}^{3,2}-B_{0}^{3,2}-B_{0}^{3,2}(\cdot+1)\right)$ it follows immediately that $\left\{\xi_{1}^{-}(\cdot-k), \xi_{2}^{-}(\cdot-k): k \in \mathbb{Z}\right\}$ is a stable basis for $S_{3,2}$.

For our further investigations we will need the cubic Hermite spline interpolant defined as

$$
\Lambda_{j}^{+} f:=\sum_{k \in \mathbb{Z}}\left(f\left(2^{-j} k\right) \xi_{1 ; j, k}^{+}+2^{-j} f^{\prime}\left(2^{-j} k\right) \xi_{2 ; j, k}^{+}\right),
$$

as well as the generalized quadratic Hermite spline interpolant

$$
\Lambda_{j}^{-} f:=\sum_{k \in \mathbb{Z}}\left(2^{j} \int_{2^{-j}(k-1)}^{2^{-j} k} f(t) d t \xi_{1 ; j, k}^{-}+f\left(2^{-j} k\right) \xi_{2 ; j, k}^{-}\right),
$$

where $\xi_{m ; j, k}^{ \pm}(x):=\xi_{m}^{ \pm}\left(2^{j} x-k\right), x \in \mathbb{R}, j, k \in \mathbb{Z}$, (i.e., $\xi_{m ; j, k}^{ \pm}$is normalized in $L_{\infty}$ ), i.e., by $f_{; j, k}$ we denote the $L_{\infty}$-normalized shifted scaling of a real-valued function $f$. Obviously, $\Lambda_{j}^{ \pm}$is a projection onto the space

$$
V_{j}^{ \pm}:=\operatorname{clos}_{L_{2}} \operatorname{span}\left\{\xi_{m ; j, k}^{ \pm}: m \in\{1,2\}, k \in \mathbb{Z}\right\}
$$

and we have for all $j \in \mathbb{Z}$,

$$
\begin{aligned}
\left(\Lambda_{j}^{ \pm} f\right)\left(2^{-j} k\right) & =f\left(2^{-j} k\right), \\
\left(\Lambda_{j}^{+} f\right)^{\prime}\left(2^{-j} k\right) & =f^{\prime}\left(2^{-j} k\right), \\
\int_{2^{-j}(k-1)}^{2^{-j} k} \Lambda_{j}^{-} f(x) d x & =\int_{2^{-j}(k-1)}^{2^{-j} k} f(x) d x, \quad k \in \mathbb{Z} .
\end{aligned}
$$

It will be convenient to express the above interpolants in terms of dual functionals

$$
\Lambda_{j}^{ \pm} f=\sum_{k \in \mathbb{Z}} \sum_{m=1}^{2}\left\langle f, \tilde{\xi}_{m ; j, k}^{ \pm}\right\rangle \xi_{m ; j, k}^{ \pm},
$$

where $\langle\cdot, \cdot\rangle$ denotes the dual pair. It is readily seen that, in the sense of distributions,

$$
\begin{array}{ll}
\tilde{\xi}_{1}^{+}=\delta_{0}, & \text { i.e., }\left\langle f, \tilde{\xi}_{1 ; j, k}^{+}\right\rangle=f\left(2^{-j} k\right), \\
\tilde{\xi}_{2}^{+}=-\delta_{0}^{\prime}, \quad \text { i.e., }\left\langle f, \tilde{\xi}_{2 ; j, k}^{+}\right\rangle=2^{-j} f^{\prime}\left(2^{-j} k\right), \\
\tilde{\xi}_{1}^{-}=\chi_{[-1,0)}, \quad \text { i.e., }\left\langle f, \tilde{\xi}_{1 ; j, k}^{-}\right\rangle=2^{j} \int_{2^{-j}(k-1)}^{2^{-j} k} f(x) d x, \\
\tilde{\xi}_{2}^{-}=\delta_{0}, \quad \text { i.e., }\left\langle f, \tilde{\xi}_{2 ; j, k}^{-}\right\rangle=f\left(2^{-j} k\right) .
\end{array}
$$

Note, that the dual functionals are $\left(L_{\infty}\right.$-normalized) scaled and shifted versions of $\tilde{\xi}_{m}^{ \pm}$, in the sense that

$$
\left\langle f, \tilde{\xi}_{m ; j, k}^{ \pm}\right\rangle=\left\langle f\left(\frac{\cdot+k}{2^{j}}\right), \tilde{\xi}_{m}^{ \pm}\right\rangle .
$$

Then, we have the following relations. 
Proposition 3.2. It holds that:

(a) $\frac{d}{d x} \tilde{\xi}_{1}^{-}=\tilde{\xi}_{1}^{+}(\cdot+1)-\tilde{\xi}_{1}^{+}, \frac{d}{d x} \tilde{\xi}_{2}^{-}=-\tilde{\xi}_{2}^{+}$in the sense of distributions.

(b) $\left\langle f^{\prime}, \tilde{\xi}_{1 ; j, k}^{-}\right\rangle=\left\langle f, 2^{j}\left(\tilde{\xi}_{1 ; j, k}^{+}-\tilde{\xi}_{1 ; j, k-1}^{+}\right)\right\rangle,\left\langle f^{\prime}, \tilde{\xi}_{2 ; j, k}^{-}\right\rangle=\left\langle f, 2^{j} \tilde{\xi}_{2 ; j, k}^{+}\right\rangle$.

(c) $\frac{d}{d x} \Lambda_{j}^{+} f=\Lambda_{j}^{-}\left(f^{\prime}\right)$, if $f \in C^{1}(\mathbb{R})$.

(d) $\left\langle\xi_{m^{\prime} ; j, k^{\prime}}^{ \pm}, \tilde{\xi}_{m ; j, k}^{ \pm}\right\rangle=\delta_{m, m^{\prime}} \delta_{k, k^{\prime}}$.

Proof. Claim (a) is obvious, and also the second part of (b). By definition, we have

$$
\begin{aligned}
\left\langle f^{\prime}, \tilde{\xi}_{1 ; j, k}^{-}\right\rangle & =2^{j} \int_{2^{-j}(k-1)}^{2^{-j} k} f^{\prime}(x) d x=2^{j}\left(f\left(2^{-j} k\right)-f\left(2^{-j}(k-1)\right)\right) \\
& =\left\langle f, 2^{j}\left(\tilde{\xi}_{1 ; j, k}^{+}-\tilde{\xi}_{1 ; j, k-1}^{+}\right)\right\rangle,
\end{aligned}
$$

which proves (b). Finally, for $f \in C^{1}(\mathbb{R})$, we have by (a), (b) and (3.7)

$$
\begin{aligned}
\frac{d}{d x} \Lambda_{j}^{+} f & =\sum_{k \in \mathbb{Z}}\left(\left\langle f, \tilde{\xi}_{1 ; j, k}^{+}\right\rangle 2^{j}\left(\xi_{1 ; j, k}^{-}-\xi_{1 ; j, k+1}^{-}\right)+\left\langle f, \tilde{\xi}_{2 ; j, k}^{+}\right\rangle 2^{j} \xi_{2 ; j, k}^{-}\right) \\
& =\sum_{k \in \mathbb{Z}}\left(\left\langle f, 2^{j}\left(\tilde{\xi}_{1 ; j, k}^{+}-\tilde{\xi}_{1 ; j, k-1}^{+}\right)\right\rangle \xi_{1 ; j, k}^{-}+\left\langle f, 2^{j} \tilde{\xi}_{2 ; j, k}^{+}\right\rangle \tilde{\xi}_{2 ; j, k}^{-}\right) \\
& =\sum_{k \in \mathbb{Z}} \sum_{m=1}^{2}\left\langle f^{\prime}, \tilde{\xi}_{m ; j, k}^{-}\right\rangle \tilde{\xi}_{i ; j, k}^{-}=\Lambda_{j}^{-} f^{\prime} .
\end{aligned}
$$

Finally (d) follows from (3.5) and (3.6).

Often, one collects the two generating functions in one vector:

$$
\boldsymbol{\xi}^{+}:=\left(\begin{array}{c}
\xi_{1}^{+} \\
\xi_{2}^{+}
\end{array}\right), \quad \boldsymbol{\xi}^{-}:=\left(\begin{array}{c}
\xi_{1}^{-} \\
\xi_{2}^{-}
\end{array}\right) .
$$

Such a vector is called a multigenerator if the integer translates form an $L_{2}$-stable basis and $\boldsymbol{\xi}$ satisfies a refinement equation

$$
\boldsymbol{\xi}(x)=\sum_{k \in \mathbb{Z}} \boldsymbol{A}_{k} \boldsymbol{\xi}(2 x-k), \quad x \in \mathbb{R} \text { a.e. },
$$

with mask $\boldsymbol{A}:=\left\{\boldsymbol{A}_{k}\right\}_{k \in \mathbb{Z}}, \boldsymbol{A}_{k} \in \mathbb{R}^{2 \times 2}$.

Applying the projectors $\Lambda_{1}^{+}$to $\xi_{i}^{+}(\dot{\overline{2}}) \in S_{4,2}, i=1,2$, it follows that $\boldsymbol{\xi}^{+}$satisfies the refinement equation (3.14) with mask matrices

$$
\boldsymbol{A}_{-1}^{+}=\left(\begin{array}{rr}
\frac{1}{2} & \frac{3}{4} \\
-\frac{1}{8} & -\frac{1}{8}
\end{array}\right), \quad \boldsymbol{A}_{0}^{+}=\left(\begin{array}{cc}
1 & 0 \\
0 & \frac{1}{2}
\end{array}\right), \quad \boldsymbol{A}_{1}^{+}=\left(\begin{array}{cc}
\frac{1}{2} & -\frac{3}{4} \\
\frac{1}{8} & -\frac{1}{8}
\end{array}\right),
$$

(see also [11, 21]) and the application of $\Lambda_{1}^{-}$to $\xi_{i}^{-}(\dot{\overline{2}}) \in S_{3,2}$ yields that $\boldsymbol{\xi}^{-}$is refinable with mask matrices

$$
\boldsymbol{A}_{-2}^{-}=\left(\begin{array}{rr}
0 & 0 \\
-\frac{1}{4} & 0
\end{array}\right), \quad \boldsymbol{A}_{-1}^{-}=\left(\begin{array}{cc}
0 & 0 \\
\frac{1}{4} & \frac{1}{4}
\end{array}\right), \quad \boldsymbol{A}_{0}^{-}=\left(\begin{array}{cc}
\frac{1}{2} & 0 \\
\frac{1}{4} & 1
\end{array}\right), \quad \boldsymbol{A}_{1}^{-}=\left(\begin{array}{rr}
\frac{1}{2} & \frac{3}{2} \\
-\frac{1}{4} & -\frac{1}{4}
\end{array}\right) .
$$


Next, we define interpolating wavelets

$$
\begin{aligned}
\eta_{m}^{+} & :=\xi_{m}^{+}(2 \cdot-1), \quad m=1,2, \\
\eta_{1}^{-} & :=\xi_{1}^{-}(2 \cdot-1)-\xi_{1}^{-}(2 \cdot-2), \quad \eta_{2}^{-}:=\xi_{2}^{-}(2 \cdot-1) .
\end{aligned}
$$

The following interpolation relations follow from (3.5) and (3.6):

$$
\begin{aligned}
\frac{d^{\nu}}{d x^{\nu}} \eta_{m}^{+}\left(\frac{k}{2}\right) & =2^{\nu} \delta_{k 1} \delta_{m-1, \nu}, \quad \nu \in\{0,1\}, \\
\eta_{m}^{-}\left(\frac{k}{2}\right) & =\delta_{k 1} \delta_{m 2}, \\
\int_{k / 2}^{(k+1) / 2} \eta_{m}^{-}(x) d x & =\frac{1}{2} \delta_{m 1}\left(\delta_{k 0}-\delta_{k 1}\right), \quad k \in \mathbb{Z}, i \in\{1,2\} .
\end{aligned}
$$

Note that we obtain the following crucial property

$$
\frac{d}{d x} \eta_{m}^{+}(x)=2 \eta_{m}^{-}(x), \quad m=1,2 .
$$

By the interpolation properties one sees immediately that the wavelet spaces

$$
W_{j}^{ \pm}:=\operatorname{clos}_{L_{2}} \operatorname{span}\left\{\eta_{m ; j, k}^{ \pm}: m \in\{1,2\}, k \in \mathbb{Z}\right\}
$$

satisfy $V_{j}^{ \pm} \cap W_{j}^{ \pm}=\{0\}$ and $V_{j}^{ \pm} \cup W_{j}^{ \pm}=V_{j+1}^{ \pm}$. In particular, we have

$$
\begin{array}{ll}
g\left(2^{-j} k\right)=g^{\prime}\left(2^{-j} k\right)=0, & \text { if } g \in W_{j}^{+}, \\
g\left(2^{-j} k\right)=\int_{2^{-j} k}^{2^{-j}(k+1)} g(x) d x=0, & \text { if } g \in W_{j}^{-},
\end{array}
$$

i.e., $\left\langle g, \tilde{\xi}_{\mu ; j, k}^{ \pm}\right\rangle=0$ and $\Lambda_{j}^{ \pm} g=0$ if $g \in W_{j}^{ \pm}$. In order to compute the wavelet expansion of a function, we are also interested in dual wavelets.

Theorem 3.3. The functionals $\tilde{\eta}_{m ; j, k}^{ \pm}$defined as $\left\langle f, \tilde{\eta}_{m ; j, k}^{ \pm}\right\rangle:=\left\langle f-\Lambda_{j}^{ \pm} f, \tilde{\xi}_{m ; j+1,2 k+1}^{ \pm}\right\rangle$ satisfy the biorthogonality conditions

$$
\begin{aligned}
\left\langle\eta_{m^{\prime} ; j^{\prime}, k^{\prime}}^{ \pm}, \tilde{\eta}_{m ; j, k}^{ \pm}\right\rangle & =\delta_{m, m^{\prime}} \delta_{j, j^{\prime}} \delta_{k, k^{\prime}} \\
\left\langle\eta_{m^{\prime} ; j, k^{\prime}}^{ \pm}, \tilde{\xi}_{m ; j, k}^{ \pm}\right\rangle & =\left\langle\xi_{m^{\prime} ; j, k^{\prime}}^{ \pm}, \tilde{\eta}_{m ; j, k}^{ \pm}\right\rangle=0
\end{aligned}
$$

for all $m, m^{\prime}=1,2$ and $j, j^{\prime}, k, k^{\prime} \in \mathbb{Z}$. Furthermore, $\tilde{\eta}_{m ; j, k}^{ \pm}$is a shifted translate of $\tilde{\eta}_{m}^{ \pm}$in the sense that

$$
\left\langle f, \tilde{\eta}_{j, k}^{ \pm}\right\rangle=\left\langle f\left(\frac{\cdot+k}{2^{j}}\right), \tilde{\eta}^{ \pm}\right\rangle
$$

where

$$
\begin{aligned}
& \tilde{\eta}_{1}^{+}:=\delta_{\frac{1}{2}}-\frac{1}{2} \delta_{0}-\frac{1}{2} \delta_{1}+\frac{1}{8} \delta_{0}^{\prime}-\frac{1}{8} \delta_{1}^{\prime}, \\
& \tilde{\eta}_{2}^{+}:=\frac{3}{4} \delta_{0}-\frac{3}{4} \delta_{1}-\frac{1}{2} \delta_{\frac{1}{2}}^{\prime}-\frac{1}{8} \delta_{0}^{\prime}-\frac{1}{8} \delta_{1}^{\prime}, \\
& \tilde{\eta}_{1}^{-}:=\chi_{\left[0, \frac{1}{2}\right]}-\chi_{\left[\frac{1}{2}, 1\right]}-\frac{1}{4} \delta_{0}+\frac{1}{4} \delta_{1}, \\
& \tilde{\eta}_{2}^{-}:=\delta_{\frac{1}{2}}+\frac{1}{4} \delta_{0}+\frac{1}{4} \delta_{1}-\frac{3}{2} \chi_{[0,1]} .
\end{aligned}
$$

Proof. The equalities in (3.17) yield $\Lambda_{j}^{ \pm} \eta_{m ; j, k}^{ \pm}=0$, i.e.,

$$
\left\langle\eta_{m^{\prime} ; j^{\prime}, k^{\prime}}^{ \pm}, \tilde{\eta}_{m ; j, k}^{ \pm}\right\rangle=\left\langle\eta_{m^{\prime} ; j^{\prime}, k^{\prime}}^{ \pm}-\Lambda_{j}^{ \pm} \eta_{m^{\prime} ; j^{\prime}, k^{\prime}}^{ \pm} \tilde{\xi}_{m ; j+1,2 k+1}^{ \pm}\right\rangle=\left\langle\eta_{m^{\prime} ; j^{\prime}, k^{\prime}}^{ \pm}, \tilde{\xi}_{m ; j+1,2 k+1}^{ \pm}\right\rangle .
$$

Now (3.18) follows from the definition of $\tilde{\eta}_{m}^{ \pm}$in (3.15) and biorthogonality for the scaling functions in Proposition 3.2(d). The equalities in (3.19) follow immediately from the definitions of $\eta_{m ; j, k}^{ \pm}$and $\tilde{\eta}_{m ; j, k}^{ \pm}$. 
Furthermore, (3.13) gives

$$
\left\langle f, \tilde{\eta}_{m ; j, k}^{ \pm}\right\rangle=\left\langle f\left(\frac{\cdot+k}{2^{j}}\right)-\Lambda_{j}^{ \pm} f\left(\frac{\cdot+k}{2^{j}}\right), \tilde{\xi}_{m ; 1,1}^{ \pm}\right\rangle .
$$

With (3.12), we obtain the representations of the particular dual functionals by

$$
\left\langle f, \tilde{\eta}_{m}^{ \pm}\right\rangle=\left\langle f, \tilde{\xi}_{m ; 1,1}^{ \pm}\right\rangle-\sum_{k \in \mathbb{Z}}\left\langle f, \tilde{\xi}_{m ; 0, k}^{ \pm}\right\rangle\left\langle\xi_{m ; 0, k}^{ \pm}, \tilde{\xi}_{m ; 1,1}^{ \pm}\right\rangle
$$

The dual functionals lead to a fast decomposition algorithm. For a given function $f=\sum_{m, k} c_{m ; j+1, k} \xi_{m ; j+1, k}^{ \pm} \in V_{j+1}^{ \pm}$we want to compute the coefficients $c_{m ; j, k}^{ \pm}$and $d_{m ; j, k}^{ \pm}$of the expansion

$$
f=\sum_{m, k} c_{m ; j, k}^{ \pm} \xi_{m ; j, k}^{ \pm}+\sum_{m, k} d_{m ; j, k}^{ \pm} \eta_{m ; j, k}^{ \pm}
$$

Applying $\tilde{\xi}_{m ; j, k}^{ \pm}$to $f$ we obtain with (3.19) that $c_{m ; j, k}^{ \pm}=\left\langle f, \tilde{\xi}_{m ; j, k}^{ \pm}\right\rangle$, i.e.,

$$
\begin{aligned}
& c_{1 ; j, k}^{+}=c_{1 ; j+1,2 k}^{+}, \quad c_{2 ; j, k}^{+}=2 c_{2 ; j+1,2 k}^{+}, \\
& c_{2 ; j, k}^{-}=c_{2 ; j+1,2 k}^{-}, \quad c_{1 ; j, k}^{-}=\frac{1}{2}\left(c_{1 ; j+1,2 k-1}^{-}+c_{1 ; j+1,2 k}^{-}\right) .
\end{aligned}
$$

Analogously, the wavelet coefficients can be obtained by $d_{m ; j, k}^{ \pm}=\left\langle f, \tilde{\eta}_{m ; j, k}^{ \pm}\right\rangle$, i.e.,

$$
\begin{aligned}
& d_{1 ; j, k}^{+}:=c_{1 ; j+1,2 k+1}^{+}-\frac{1}{2} c_{1 ; j+1,2 k}^{+}-\frac{1}{2} c_{1 ; j+1,2 k+2}^{+}-\frac{1}{4} c_{2 ; j+1,2 k}^{+}+\frac{1}{4} c_{2 ; j+1,2 k+2}^{+}, \\
& d_{2 ; j, k}^{+}:=\frac{3}{4} c_{1 ; j+1,2 k}^{+}-\frac{3}{4} c_{1 ; j+1,2 k+2}^{+}+c_{2 ; j+1,2 k+1}^{+}+\frac{1}{4} c_{2 ; j+1,2 k}^{+}+\frac{1}{4} c_{2 ; j+1,2 k+2}^{+}, \\
& d_{1 ; j, k}^{-}:=\frac{1}{2} c_{1 ; j+1,2 k+1}^{-}-\frac{1}{2} c_{1 ; j+1,2 k+2}^{-}-\frac{1}{4} c_{2 ; j+1,2 k}^{-}+\frac{1}{4} c_{2 ; j+1,2 k+2}^{-}, \\
& d_{2 ; j, k}^{-}:=c_{2 ; j+1,2 k+1}^{-}+\frac{1}{4} c_{2 ; j+1,2 k}^{-}+\frac{1}{4} c_{2 ; j+1,2 k+2}^{-}-\frac{3}{4} c_{1 ; j+1,2 k+1}^{-}-\frac{3}{4} c_{1 ; j+1,2 k+2}^{-} .
\end{aligned}
$$

Finally, relation (3.16) implies an analogous relation $\frac{d}{d x} \tilde{\eta}_{m}^{-}=-\frac{1}{2} \tilde{\eta}_{m}^{+}$for the duals in the sense of distributions, i.e.,

$$
\left\langle f^{\prime}, \tilde{\eta}_{m ; j, k}^{-}\right\rangle=-2^{j-1}\left\langle f, \tilde{\eta}_{m ; j, k}^{+}\right\rangle .
$$

3.2. Characterization of function spaces. For any function $f \in C^{1}(\mathbb{R})(f \in$ $C(\mathbb{R}))$ we can compute the coefficients $\left\langle f, \tilde{\eta}_{m ; j, k}^{+}\right\rangle\left(\left\langle f, \tilde{\eta}_{m ; j, k}^{-}\right\rangle\right)$. An interesting question is, in which sense the expansion $\sum_{j, k}\left\langle f, \tilde{\eta}_{m ; j, k}^{ \pm}\right\rangle \eta_{m ; j, k}^{ \pm}$is to be understood. In this section we show that Hermite interpolatory wavelets form unconditional bases for certain Besov spaces, which are contained in the space of continuous (differentiable) functions.

In the sequel we will use the following standard definition of Besov spaces. For $0<p, q \leq \infty$ and $s>0$ we define a semi-norm

$$
|f|_{B_{q}^{s}\left(L_{p}(\Omega)\right)}:= \begin{cases}\left(\sum_{j \in \mathbb{Z}} 2^{j q s} \omega_{m}\left(f, 2^{-j}, L_{p}(\Omega)\right)^{q}\right)^{\frac{1}{q}}, & \text { if } 1 \leq q<\infty, \\ \sup _{j \in \mathbb{Z})} 2^{j s} \omega_{d}\left(f, 2^{-j}, L_{p}(\Omega)\right), & \text { if } q=\infty,\end{cases}
$$

with $m:=\lfloor s\rfloor+1$, where $\omega_{m}\left(f, t, L_{p}(\Omega)\right)$ is the $m$-th order modulus of smoothness. The Besov space $B_{p, q}^{s}(\Omega)$ is the set of all $f \in L_{p}(\Omega)$ such that $|f|_{B_{q}^{s}\left(L_{p}(\Omega)\right)}$ is finite. A norm is defined by

$$
\|f\|_{B_{q}^{s}\left(L_{p}(\Omega)\right)}:=\|f\|_{L_{p}(\Omega)}+|f|_{B_{q}^{s}\left(L_{p}(\Omega)\right)} .
$$


There are numerous equivalent norms in the literature. Here, we only need that an equivalent norm is obtained if $m$ in (3.23) is replaced by any integer $d>s$ (see e.g. 9]).

Furthermore, we need the following discrete norms for coefficient sequences. For the sequences $\boldsymbol{\beta}=\left(\beta_{k}\right)_{k \in \mathbb{Z}^{n}}, \boldsymbol{\alpha}=\left(\alpha_{j k}\right)_{j \geq j_{0}, k \in \mathbb{Z}^{n}}$, and $\boldsymbol{\alpha}_{j}=\left(\alpha_{j k}\right)_{k \in \mathbb{Z}^{n}}$, we define

$$
\begin{aligned}
\|\boldsymbol{\beta}\|_{\ell_{p}} & := \begin{cases}\left(\sum_{k}\left|\beta_{k}\right|^{p}\right)^{\frac{1}{p}}, & \text { if } 0<p<\infty, \\
\sup _{k}\left|\beta_{k}\right|, & \text { if } p=\infty,\end{cases} \\
\|\boldsymbol{\alpha}\|_{\ell_{p q}^{s}} & := \begin{cases}\left(\sum_{j}\left(2^{j\left(s-\frac{n}{p}\right)}\left\|\boldsymbol{\alpha}_{j}\right\|_{\ell_{p}}\right)^{q}\right)^{\frac{1}{q}}, & \text { if } 0<q<\infty, \\
\sup _{j} 2^{j s}\left\|\boldsymbol{\alpha}_{j}\right\|_{\ell_{p}}, & \text { if } q=\infty,\end{cases}
\end{aligned}
$$

for $p \in(0, \infty]$. In [16, Besov spaces on the real line are characterized in terms of the wavelet expansion with respect to interpolatory wavelets (see Theorem 2.1). Here we need more general results for the characterization of Besov spaces on $\mathbb{R}^{n}$ with Hermite interpolatory wavelets. Our characterization is essentially based on results reported in 9]. Even though, the following results are not reported in the literature in the presented form, the techniques used therein are more or less standard. Hence, we collected the proofs in Appendix A

The Besov norm of a multiscale representation can be estimated by the corresponding discrete norm of the coefficients.

Lemma 3.4. If $\phi \in B_{\infty}^{\sigma}\left(L_{p}\left(\mathbb{R}^{n}\right)\right)$ is compactly supported, $0<p, q \leq \infty$, and $0<s<\sigma$, then

$$
\begin{aligned}
\left\|\sum_{k \in \mathbb{Z}^{n}} \beta_{k} \phi\left(2^{j} \cdot-k\right)\right\|_{B_{q}^{s}\left(L_{p}\left(\mathbb{R}^{n}\right)\right)} & \lesssim 2^{\left(s-\frac{n}{p}\right) j}\|\boldsymbol{\beta}\|_{\ell_{p}}, \\
\left\|\sum_{j=j_{0}}^{\infty} \sum_{k \in \mathbb{Z}^{n}} \alpha_{j, k} \phi\left(2^{j} \cdot-k\right)\right\|_{B_{q}^{s}\left(L_{p}\left(\mathbb{R}^{n}\right)\right)} & \lesssim\|\boldsymbol{\alpha}\|_{\ell_{p q}^{s}} .
\end{aligned}
$$

Applying Lemma 3.4 to our interpolatory wavelets we obtain by the facts $\xi_{m}^{+} \in$ $B_{\infty}^{2+1 / p}\left(L_{p}(\mathbb{R})\right)$ and $\xi_{m}^{-} \in B_{\infty}^{1+1 / p}\left(L_{p}(\mathbb{R})\right)$ the following corollary.

Corollary 3.5. Let $0<s<2+\frac{1}{p}$ and $0<p, q \leq \infty$. Then

$$
\left\|\sum_{m=1}^{2}\left(\sum_{k \in \mathbb{Z}} \beta_{m, k} \xi_{m ; j_{0}, k}^{+}+\sum_{j=j_{0}}^{\infty} \sum_{k \in \mathbb{Z}} \alpha_{m ; j, k} \eta_{m ; j, k}^{+}\right)\right\|_{B_{q}^{s}\left(L_{p}(\mathbb{R})\right)} \lesssim \sum_{m=1}^{2}\left(\left\|\boldsymbol{\beta}_{m}\right\|_{\ell_{p}}+\left\|\boldsymbol{\alpha}_{m}\right\|_{\ell_{p q}^{s}}\right) .
$$

If $0<s<1+\frac{1}{p}$, the estimate also holds if $\xi_{m}^{+}$and $\eta_{m}^{+}$are replaced by $\xi_{m}^{-}$and $\eta_{m}^{-}$, respectively.

Note, that the upper bounds for $s$ are sharp, since for $s>2+\frac{1}{p}$ and $s>1+\frac{1}{p}$, e.g., $\left\|\eta_{m ; j, k}^{ \pm}\right\|_{B_{q}^{s}\left(L_{p}(\mathbb{R})\right)}$ is unbounded. In order to estimate the Besov norm of a function by a discrete norm of its wavelet coefficients we will employ the vanishing moments of the dual functionals, which enable us to estimate the wavelet coefficients by the error of a locally best approximation

$$
E_{d}\left(f, W_{\tau}^{\mu}(\sigma)\right):=\inf _{P \in \Pi_{d-1}}\|f-P\|_{W_{\tau}^{\mu}(\sigma)}
$$

by a polynomial $P$ of degree less than $d$, in the norm of the Sobolev space $W_{\tau}^{\mu}(\sigma)$ on some $\sigma \subset \mathbb{R}^{n}$. These errors depend in turn on the Besov regularity of $f$. 
Lemma 3.6. Let $\frac{n}{p}-\frac{n}{\tau}+\mu<s<d, s>0, \mu \in \mathbb{N}_{0}, 0<p, q \leq \infty, 1 \leq \tau \leq \infty$. Furthermore, let $\sigma_{j k} \subset 2^{-j}(\sigma+k), j \in \mathbb{Z}, k \in \mathbb{Z}^{n}$, with a cube $\sigma \subset \mathbb{R}^{n}$. Then

$$
\sum_{j \in \mathbb{Z}}\left(2^{j\left(s-\frac{n}{p}+\frac{n}{\tau}-|\mu|\right)}\left(\sum_{k \in \mathbb{Z}^{n}} E_{d}\left(f, W_{\tau}^{\mu}\left(\sigma_{j k}\right)\right)^{p}\right)^{\frac{1}{p}}\right)^{q} \lesssim|f|_{B_{q}^{s}\left(L_{p}\left(\mathbb{R}^{n}\right)\right)}^{q} .
$$

We will also need an estimate for the coefficients of the scaling functions on the coarse level. This estimate will be based on the following lemma.

Lemma 3.7. Let $\frac{1}{p}-\frac{1}{\tau}+\mu<s, \mu \in \mathbb{N}_{0}, 0<p, q \leq \infty, 1 \leq \tau \leq \infty$. For $\sigma_{k} \subset \sigma+k$, $k \in \mathbb{Z}^{n}$, where $\sigma \subset \mathbb{R}^{n}$ is an n-dimensional cube, we have

$$
\left(\sum_{k \in \mathbb{Z}^{n}}\|f\|_{W_{\tau}^{\mu}\left(\sigma_{k}\right)}^{p}\right)^{\frac{1}{p}} \lesssim\|f\|_{B_{q}^{s}\left(L_{p}\left(\mathbb{R}^{n}\right)\right)} .
$$

For our Hermite spline wavelets we obtain from Lemma 3.6 and Lemma 3.7 the following estimate.

Corollary 3.8. Let $f \in B_{q}^{s}\left(L_{p}(\mathbb{R})\right), 1+\frac{1}{p}<s<4,0<p, q \leq \infty$. Then the coefficient sequences $\boldsymbol{\alpha}_{m}:=\left(\left\langle f, \tilde{\eta}_{m ; j, k}^{+}\right\rangle\right)_{j \geq j_{0}, k \in \mathbb{Z}}, \boldsymbol{\beta}_{m}:=\left(\left\langle f, \tilde{\xi}_{m ; j_{0}, k}^{+}\right\rangle\right)_{k \in \mathbb{Z}}, j_{0} \in \mathbb{Z}$, $m=1,2$, satisfy

$$
\sum_{m=1}^{2}\left(\left\|\boldsymbol{\beta}_{m}\right\|_{\ell_{p}}+\left\|\boldsymbol{\alpha}_{m}\right\|_{\ell_{p q}^{s}}\right) \lesssim\|f\|_{B_{q}^{s}\left(L_{p}(\mathbb{R})\right)}
$$

If $\frac{1}{p}<s<3$, the estimate holds also if $\tilde{\xi}_{m}^{+}$and $\tilde{\eta}_{m}^{+}$are replaced by $\tilde{\xi}_{m}^{-}$and $\tilde{\eta}_{m}^{-}$.

Again, the bounds for $s$ are sharp. The lower bounds are necessary for the imbedding of the Besov space in $C^{1}(\mathbb{R})$ or $C^{0}(\mathbb{R})$, which is in turn needed for the coefficents to be well defined. On the other hand, the upper bound for $s$ is imposed by the maximal number of vanishing moments of the dual wavelets, i.e., by the polynomial exactness of the corresponding spline spaces. Finally, putting all of the pieces together, we obtain that these wavelets form unconditional bases for a certain range of Besov spaces.

Theorem 3.9. Let $1+\frac{1}{p}<s<\min \left(4,2+\frac{1}{p}\right), 0<p, q \leq \infty$. Then every $f \in B_{q}^{s}\left(L_{p}(\mathbb{R})\right)$ has the expansion

$$
f=\sum_{m=1}^{2}\left(\sum_{k \in \mathbb{Z}} \beta_{m, k} \xi_{j_{0}, k}^{+}+\sum_{j=j_{0}}^{\infty} \sum_{k \in \mathbb{Z}} \alpha_{m ; j, k} \eta_{m ; j, k}^{+}\right)
$$

with uniquely determined coefficients $\beta_{m, k}=\left\langle f, \tilde{\xi}_{m ; j_{0}, k}^{+}\right\rangle$and $\alpha_{m, j, k}=\left\langle f, \tilde{\eta}_{m ; j, k}^{+}\right\rangle$. Furthermore,

$$
\|f\|_{B_{q}^{s}\left(L_{p}(\mathbb{R})\right)} \sim \sum_{m=1}^{2}\left(\left\|\boldsymbol{\beta}_{m}\right\|_{\ell_{p}}+\left\|\boldsymbol{\alpha}_{m}\right\|_{\ell_{p q}^{s}}\right) .
$$

If $\xi_{m}^{+}, \eta_{m}^{+}$are replaced by $\xi_{m}^{-}, \eta_{m}^{-}$, then the result holds for $\frac{1}{p}<s<\min \left(3,1+\frac{1}{p}\right)$.

Proof. The unconditional convergence of (3.26) is shown with the estimates in Corollaries 3.5 and 3.8 and the biorthogonality relations in Proposition 3.2(d), (3.18), and (3.19). Then the norm equivalence follows immediately from Corollaries 3.5 and 3.8 . 
Note that the bounds for $s$ are relatively narrow. In particular, we cannot choose the same value of $s$ for the wavelets $\eta_{m}^{-}$and $\eta_{m}^{+}$. This will cause some difficulties for the divergence-free wavelets constructed in Section 3.3 below, which can be avoided by considering anisotropic Besov spaces as is done later in Section 3.4. However, wavelets of higher regularity might be desirable in some cases.

3.3. Divergence-free interpolatory Hermite splines. We now describe a construction of divergence-free interpolatory Hermite spline wavelets based on (3.7). We first consider the interpolatory tensor-product MRA $V_{j}^{I}$ similar to (2.12). Note that these spaces are generated by scaled translates of

$$
\varphi_{m}^{I}\left(x_{1}, \ldots, x_{n}\right):=\prod_{\nu=1}^{n} \xi_{m_{\nu}, \nu}^{I}\left(x_{\nu}\right), \quad m=\left(m_{1}, \ldots, m_{n}\right)^{T} \in\{1,2\}^{n}
$$

where

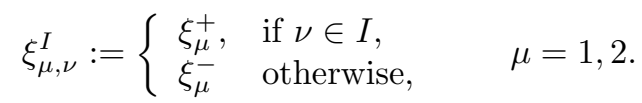

This means that $V_{j}^{I}$ is generated by a multigenerator of dimension $2^{n}$. The corresponding wavelets are

$$
\psi_{e, m}^{I}\left(x_{1}, \ldots, x_{n}\right):=\prod_{\nu=1}^{n} \vartheta_{e_{\nu}, m_{\nu}, \nu}^{I}\left(x_{\nu}\right), \quad e \in E_{n}^{*}, m=\left(m_{1}, \ldots, m_{n}\right)^{T} \in\{1,2\}^{n},
$$

where

$$
\vartheta_{\ell, \mu, \nu}^{I}:= \begin{cases}\xi_{\mu, \nu}^{I}, & \text { if } \ell=0 \\ \eta_{\mu, \nu}^{I} & \text { otherwise }\end{cases}
$$

and $\eta_{i, \nu}^{I}$ is defined in a similar fashion as $\xi_{i, \nu}^{I}$. Analogously, we define $\tilde{\varphi}_{m}^{I}$ and $\tilde{\psi}_{e, m}^{I}$ as tensor product functionals. Thus, we obtain $\left|E_{n}^{*}\right| 2^{n}=\left(2^{n}-1\right) 2^{n}$ wavelets, i.e., 12 wavelets in $2 \mathrm{D}$ and 56 wavelets in $3 \mathrm{D}$. We denote by

$$
\Lambda_{j}^{I}:=\bigotimes_{\nu=1}^{n} \Lambda_{\nu ; j}, \quad \Lambda_{\nu ; j}:= \begin{cases}\Lambda_{j}^{+}, & \text {if } \nu \in I, \\ \Lambda_{j}^{-}, & \text {if } \nu \notin I,\end{cases}
$$

the corresponding Hermite spline interpolants. Before we proceed, we collect some properties of these interpolants.

Proposition 3.10. The following properties hold for sufficiently smooth functions $f: \mathbb{R}^{n} \rightarrow \mathbb{R}:$

(a) $\left\langle\Lambda_{j}^{I} f, \tilde{\varphi}_{m ; j, k}^{I}\right\rangle=\left\langle f, \tilde{\varphi}_{m ; j, k}^{I}\right\rangle, \quad$ for $k \in \mathbb{Z}^{n}, m \in\{1,2\}^{n}$;

(b) $\frac{\partial}{\partial x_{i}} \Lambda_{j}^{I} f=\Lambda_{j}^{I \backslash\{i\}}\left(\frac{\partial}{\partial x_{i}} f\right)$, if $i \in I$;

(c) $\frac{\partial}{\partial x_{i}} \psi_{e, m}^{I}=2 \psi_{e, m}^{I \backslash\{i\}}$, if $i \in I, e_{i}=1$.

Proof. Task (a) follows directly from (3.9) -3.11), whereas (b) follows from Proposition 3.2 (c). As for (c), by $i \in I$ and $e_{i}=1$ we have $\vartheta_{e_{i}, m_{i}, i}^{I}=\vartheta_{1, m_{i}, i}^{I}=\eta_{m_{i}}^{+}$so that the claim follows from (3.16).

Note that (a) describes the (Hermite) interpolation properties of $\Lambda_{j}^{I}$, similarly to (3.9) - (3.11) in the univariate setting. In particular, for $m_{i}=1, i \in I$, and $m_{i}=2$, 
$i \notin I$, statement (a) becomes $\Lambda_{j}^{I} f\left(2^{-j} k\right)=f\left(2^{-j} k\right)$. The next step is again a vector-valued MRA for the component $i$ similar to (2.14), i.e.,

$$
\boldsymbol{\varphi}_{m, i}:=\varphi_{m}^{\{i\}} \boldsymbol{\delta}_{i}, \quad m \in\{1,2\}^{n}, 1 \leq i \leq n,
$$

and the corresponding interpolants

$$
\boldsymbol{\Lambda}_{i ; j}:=\Lambda_{j}^{\{i\}} \boldsymbol{\delta}_{i} .
$$

Finally, we obtain an interpolatory MRA $\left\{\boldsymbol{V}_{j}\right\}_{j \geq j_{0}}$ in $\boldsymbol{C}\left(\mathbb{R}^{n}\right)$ generated by

$$
\left\{\boldsymbol{\varphi}_{m, i}: m \in\{1,2\}^{n}, 1 \leq i \leq n\right\}
$$

with the associated interpolant $\boldsymbol{\Lambda}_{j}:=\sum_{i=1}^{n} \boldsymbol{\Lambda}_{i ; j}$. Hence, we have $n 2^{n}$ generators. As an immediate consequence of Proposition 3.10 we obtain

Proposition 3.11. For a vector field $\boldsymbol{f} \in \boldsymbol{C}\left(\operatorname{div} ; \mathbb{R}^{n}\right)$ we have

$$
\operatorname{div}\left(\boldsymbol{\Lambda}_{j} \boldsymbol{f}\right)=\Lambda_{j}^{\emptyset}(\operatorname{div} \boldsymbol{f}),
$$

where $\Lambda_{j}^{\emptyset}:=\Lambda_{j}^{-} \otimes \cdots \otimes \Lambda_{j}^{-}$.

Proof. With Proposition 3.10(b), we obtain

$$
\operatorname{div}\left(\boldsymbol{\Lambda}_{j} \boldsymbol{f}\right)=\sum_{i=1}^{n} \frac{\partial}{\partial x_{i}} \Lambda_{j}^{\{i\}} f_{i}=\sum_{i=1}^{n} \Lambda_{j}^{\emptyset}\left(\frac{\partial}{\partial x_{i}} f_{i}\right)=\Lambda_{j}^{\emptyset}(\operatorname{div} \boldsymbol{f}) .
$$

The Hermite interpolant $\boldsymbol{\Lambda}_{j}$ is used to interpolate given sample data of a flow field. The above proposition ensures that the interpolant of an incompressible flow field remains divergence-free.

Now, we construct divergence-free interpolatory multiwavelets. Let us first briefly sketch the idea, which is similar to (2.16) above. Let $\boldsymbol{W}_{j}:=\boldsymbol{V}_{j+1} \ominus \boldsymbol{V}_{j}$ denote the vector-valued interpolatory wavelet spaces. The road map is to decompose $\boldsymbol{W}_{j}$ as $\boldsymbol{W}_{j}^{\nabla}:=\boldsymbol{W}_{j} \cap \boldsymbol{N}(\operatorname{div} ; \Omega), \boldsymbol{W}_{j}^{\Delta}:=\boldsymbol{W}_{j} \ominus \boldsymbol{W}_{j}^{\nabla}$ in a stable way. To this end, we define $\boldsymbol{\psi}_{e, m, i}:=\psi_{e, m}^{\{i\}} \boldsymbol{\delta}_{i}$ so that

$$
\boldsymbol{W}_{j}=\operatorname{clos} \operatorname{span}\left\{\boldsymbol{\psi}_{e, m, i ; j, k}: e \in E_{n}^{*}, m \in\{1,2\}^{n}, i=1, \ldots, n, k \in \mathbb{Z}^{n}\right\} .
$$

This means, we have

$$
\left|E_{n}^{*}\right| 2^{n} n=n 2^{n}\left(2^{n}-1\right)
$$

vector-wavelets, i.e., 24 in $2 \mathrm{D}$ and 168 in $3 \mathrm{D}$. From (3.16) we conclude that

$$
\frac{\partial}{\partial x_{i}} \psi_{e, m, i: i}=2 \psi_{e, m}^{\emptyset}, \quad \text { if } e_{i}=1 \text {. }
$$

For $e \in E_{n}^{*}$ and $i \neq i_{e}$, we define

$$
\boldsymbol{\psi}_{e, m, i}^{\nabla}:=\psi_{e, m}^{\{i\}} \boldsymbol{\delta}_{i}-\frac{1}{2} \frac{\partial}{\partial x_{i}} \psi_{e, m}^{\left\{i, i_{e}\right\}} \boldsymbol{\delta}_{i_{e}} .
$$

Again, it is readily seen that due to $e_{i_{e}}=1$, we have

$$
\operatorname{div} \boldsymbol{\psi}_{e, m, i}^{\nabla}=\frac{\partial}{\partial x_{i}} \psi_{e, m}^{\{i\}}-\frac{1}{2} \frac{\partial}{\partial x_{i_{e}}} \frac{\partial}{\partial x_{i}} \psi_{e, m}^{\left\{i, i_{e}\right\}}=\frac{\partial}{\partial x_{i}} \psi_{e, m}^{\{i\}}-\frac{\partial}{\partial x_{i}} \psi_{e, m}^{\{i\}}=0 .
$$

In order to obtain the desired decomposition, we define

$$
\boldsymbol{\psi}_{e, m}^{\Delta}:=\psi_{e, m}^{\left\{i_{e}\right\}} \boldsymbol{\delta}_{i_{e}} .
$$

The dual functionals for the divergence-free wavelets turn out to be the standard ones, i.e.,

$$
\tilde{\boldsymbol{\psi}}_{e, m, i}^{\nabla}:=\tilde{\psi}_{e, m}^{\{i\}} \boldsymbol{\delta}_{i}, \quad i \neq i_{e}
$$



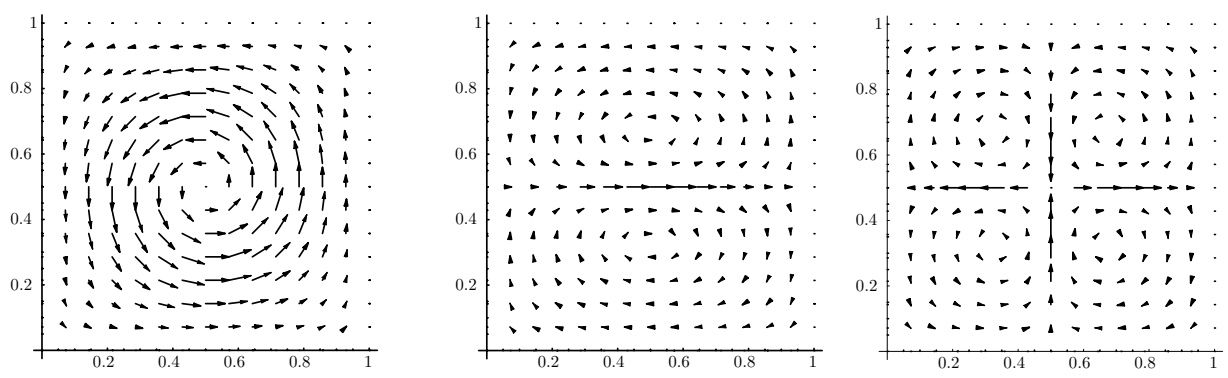

FIGURE 2. Interpolatory divergence-free wavelets $\psi_{e, m}^{\Delta}$ according to $e=(1,1), i=1$ and the choices (from left to right) $m=(1,1)$, $m=(1,2)$ and $m=(2,2)$.

Furthermore, it is easy to verify that $\tilde{\boldsymbol{\psi}}_{e, m}^{\Delta}:=-\frac{1}{2} \operatorname{grad} \tilde{\psi}_{e, m}^{\emptyset}$, where the derivatives are meant in the sense of distributions (cf. (3.22) ), i.e.,

$$
\left\langle\boldsymbol{f}, \tilde{\boldsymbol{\psi}}_{e, m}^{\Delta}\right\rangle=-\frac{1}{2}\left\langle\boldsymbol{f}, \operatorname{grad} \tilde{\psi}_{e, m}^{\emptyset}\right\rangle=\frac{1}{2}\left\langle\operatorname{div} \boldsymbol{f}, \tilde{\psi}_{e, m}^{\emptyset}\right\rangle .
$$

In Figure 2, we have depicted some divergence-free vector wavelets.

3.4. Characterization of spaces of vector fields. We will consider the spaces

$$
\check{\boldsymbol{B}}_{q}^{s}\left(\boldsymbol{L}_{p}\left(\mathbb{R}^{n}\right)\right):=\check{\boldsymbol{B}}_{q, p}^{s}:=\left\{\boldsymbol{f} \in \boldsymbol{B}_{q}^{s}\left(\boldsymbol{L}_{p}\left(\mathbb{R}^{n}\right)\right): \frac{\partial}{\partial x_{i}} f_{i} \in B_{q}^{s}\left(L_{p}\left(\mathbb{R}^{n}\right)\right), i=1, \ldots, n\right\}
$$

with the semi-norm

$$
|\boldsymbol{f}|_{\check{\boldsymbol{B}}_{q, p}^{s}}:=\max _{i}\left|\frac{\partial}{\partial x_{i}} f_{i}\right|_{B_{q}^{s}\left(L_{p}\left(\mathbb{R}^{n}\right)\right)}
$$

and the norm $\|\boldsymbol{f}\|_{\check{\boldsymbol{B}}_{q, p}^{s}}:=\|\boldsymbol{f}\|_{\boldsymbol{B}_{q}^{s}\left(\boldsymbol{L}_{p}\left(\mathbb{R}^{n}\right)\right)}+|\boldsymbol{f}|_{\check{\boldsymbol{B}}_{q, p}^{s}}$. Note that equivalent norms can be defined by replacing the maximum by any $p$-norm for $\mathbb{R}^{n}$.

The components of a vector field $\boldsymbol{f} \in \check{\boldsymbol{B}}_{q}^{s}\left(\boldsymbol{L}_{p}\left(\mathbb{R}^{n}\right)\right)$ are from certain anisotropic Besov spaces (see [5]). In particular, we have for $\boldsymbol{f} \in \check{\boldsymbol{B}}_{q}^{s}\left(\boldsymbol{L}_{p}\left(\mathbb{R}^{n}\right)\right)$ that $\operatorname{div} \boldsymbol{f} \in$ $B_{q}^{s}\left(L_{p}\left(\mathbb{R}^{n}\right)\right)$.

Theorem 3.12. Let $0<s<1+\frac{1}{p}, 0<p, q \leq \infty$ and set

$$
\boldsymbol{g}:=\sum_{j \geq j_{0}} \sum_{e, m, k}\left(\sum_{i \neq i_{e}} \alpha_{e, m, i ; j, k}^{\nabla} \boldsymbol{\psi}_{e, m, i ; j, k}^{\nabla}+\alpha_{e, m ; j, k}^{\Delta} \boldsymbol{\psi}_{e, m ; j, k}^{\Delta}\right) .
$$

Then, we have

$$
\left\|\sum_{m, i, k} \beta_{m, i ; k} \boldsymbol{\varphi}_{m, i ; j_{0}, k}+\boldsymbol{g}\right\|_{\check{\boldsymbol{B}}_{q, p}^{s}} \lesssim \sum_{m, i}\left\|\boldsymbol{\beta}_{m, i}\right\|_{\ell_{p}}+\sum_{e, m}\left(\left\|\boldsymbol{\alpha}_{e, m}^{\Delta}\right\|_{\ell_{p q}^{s+1}}+\sum_{i \neq i_{e}}\left\|\boldsymbol{\alpha}_{e, m, i}^{\nabla}\right\|_{\ell_{p q}^{s+1}}\right) .
$$

Proof. Let us choose a fixed but arbitrary $\nu$. From the construction of the wavelets we know that the $\nu$-th component has the form

$$
g_{\nu}=\sum_{\kappa} \sum_{j \geq j_{0}} \sum_{k \in \mathbb{Z}^{n}} \alpha_{\kappa, j, k} \Phi_{\nu, \kappa}\left(2^{j} \cdot-k\right),
$$

where $\kappa$ represents any possible choice of $e, m, i$ and $\Delta$ or $\nabla$. Furthermore,

$$
\Phi_{\nu, \kappa}=\bigotimes_{\ell=1}^{\infty} \phi_{\nu, \kappa, \ell}
$$


with compactly supported Hermite splines $\phi_{\nu, \kappa, \ell} \in S_{3,2}$ for $\nu \neq \ell$ and $\phi_{\nu, \kappa, \nu} \in S_{4,2}$. Then

$$
\frac{\partial}{\partial x_{\nu}} g_{\nu}=\sum_{\kappa} \sum_{j \geq j_{0}} \sum_{k \in \mathbb{Z}^{n}} 2^{j} \alpha_{\kappa, j, k}\left(\frac{\partial}{\partial x_{\nu}} \Phi_{\nu, \kappa}\right)\left(2^{j} \cdot-k\right),
$$

where $\frac{\partial}{\partial x_{\nu}} \Phi_{\nu, \kappa}$ is a tensor product of compactly supported splines from $S_{3,2}$ and therefore contained in $B_{\infty}^{1+\frac{1}{p}}\left(L_{p}\left(\mathbb{R}^{n}\right)\right)$. Now, Lemma 3.4 yields

$$
\left\|\frac{\partial}{\partial x_{\nu}} g_{\nu}\right\|_{B_{q}^{s}\left(L_{p}\left(\mathbb{R}^{n}\right)\right)} \lesssim \sum_{\kappa}\left\|\boldsymbol{\alpha}_{\kappa}\right\|_{\ell_{p q}^{s+1}} .
$$

Hence,

$$
\|\boldsymbol{g}\|_{\check{\boldsymbol{B}}_{q, p}^{s}} \lesssim \sum_{e, m}\left(\left\|\boldsymbol{\alpha}_{e, m}^{\Delta}\right\|_{\ell_{p q}^{s+1}}+\sum_{i \neq i_{e}}\left\|\boldsymbol{\alpha}_{e, m, i}^{\nabla}\right\|_{\ell_{p q}^{s+1}}\right)
$$

The estimate

$$
\left\|\sum_{m, i, k} \beta_{m, i, k} \boldsymbol{\varphi}_{m, i ; j_{0}, k}\right\|_{\check{\boldsymbol{B}}_{q, p}^{s}} \lesssim \sum_{m, i}\left\|\boldsymbol{\beta}_{m, i}\right\|_{\ell_{p}}
$$

follows analogously.

Theorem 3.13. Let $\frac{n}{p}<s<3,0<p, q \leq \infty$.

Then the sequences $\boldsymbol{\beta}_{m, i}:=\left(\left\langle\boldsymbol{f}, \tilde{\boldsymbol{\varphi}}_{m, i ; j_{0}, k}\right\rangle\right)_{k \in \mathbb{Z}}, \boldsymbol{\alpha}_{e, m, i}^{\nabla}:=\left(\left\langle\boldsymbol{f}, \tilde{\boldsymbol{\psi}}_{e, m, i ; j, k}^{\nabla}\right\rangle\right)_{j \geq j_{0}, k \in \mathbb{Z}}$ and $\boldsymbol{\alpha}_{e, m}^{\Delta}:=\left(\left\langle\boldsymbol{f}, \tilde{\boldsymbol{\psi}}_{e, m ; j, k}^{\Delta}\right\rangle\right)_{j \geq j_{0}, k \in \mathbb{Z}}, j_{0} \in \mathbb{Z}$, satisfy

$$
\sum_{m, i}\left\|\boldsymbol{\beta}_{m, i}\right\|_{\ell_{p}}+\sum_{e, m}\left(\left\|\boldsymbol{\alpha}_{e, m}^{\Delta}\right\|_{\ell_{p q}^{s+1}}+\sum_{i \neq i_{e}}\left\|\boldsymbol{\alpha}_{e, m, i}^{\nabla}\right\|_{\ell_{p q}^{s+1}}\right) \lesssim\|\boldsymbol{f}\|_{\check{B}_{q, p}^{s}}
$$

Proof. If $e_{i}=1$, we have by (3.22) that

$$
\begin{aligned}
\alpha_{e, m, i ; j, k}^{\nabla}=\left\langle\boldsymbol{f}, \tilde{\boldsymbol{\psi}}_{e, m, i ; j, k}^{\nabla}\right\rangle=\left\langle f_{i}, \tilde{\psi}_{e, m ; j, k}^{\{i\}}\right\rangle & =2^{-j-1}\left\langle\frac{\partial}{\partial x_{i}} f_{i}, \tilde{\psi}_{e, m ; j, k}^{\emptyset}\right\rangle \\
& \lesssim 2^{-j-1} E_{3}\left(\frac{\partial}{\partial x_{i}} f_{i}, L_{\infty}\left(\sigma_{j k}\right)\right),
\end{aligned}
$$

where $\sigma_{j k}=2^{-j}\left([0,1]^{d}+k\right)$. Now the application of Lemma 3.6 yields

$$
\left\|\boldsymbol{\alpha}_{e, m, i}^{\nabla}\right\|_{\ell_{p q}^{s+1}} \lesssim\left|\frac{\partial f_{i}}{\partial x_{i}}\right|_{B_{q}^{s}\left(L_{p}\left(\mathbb{R}^{n}\right)\right)} \lesssim\|\boldsymbol{f}\|_{\check{\boldsymbol{B}}_{q, p}^{s}} .
$$

The same estimate follows for $e_{i}=0$ and $m_{i}=2$ by Proposition 3.2. On the other hand, for $e_{i}=0$ and $m_{i}=1$, we assume w.l.o.g. that $i=1$ and then

$$
\left\langle\boldsymbol{f}, \tilde{\boldsymbol{\psi}}_{e, m, 1 ; j, k}^{\nabla}\right\rangle=\left\langle f_{1}, \tilde{\psi}_{e, m ; j, k}^{\{1\}}\right\rangle=\left\langle f_{1}, \delta_{2^{-j} k_{1}} \otimes \vartheta_{e_{2}, m_{2}}^{-} \otimes \ldots \otimes \vartheta_{e_{n}, m_{n}}^{-}\right\rangle .
$$

Now, set $X:=\left\{g \in C^{1}\left(\mathbb{R}^{n}\right):\left.g\right|_{x_{1}=2^{-j} k_{1}} \in \Pi_{3}\right\}$. Obviously,

$$
\left\langle g, \tilde{\psi}_{e, m ; j, k}^{\{1\}}\right\rangle=\left\langle\left. g\right|_{x_{1}=2^{-j} k_{1}}, \vartheta_{e_{2}, m_{2}}^{-} \otimes \ldots \otimes \vartheta_{e_{n}, m_{n}}^{-}\right\rangle=0, \quad g \in X
$$


Hence, we have with Proposition 3.2 ,

$$
\begin{aligned}
\left|\left\langle f_{1}, \tilde{\psi}_{e, m ; j, k}^{\{1\}}\right\rangle\right| & =\inf _{g \in X}\left|\left\langle f_{1}-g, \tilde{\psi}_{e, m ; j, k}^{\{1\}}\right\rangle\right| \\
\leq & \inf _{g \in X}\left(2^{-j}\left|\left\langle\frac{\partial}{\partial x_{1}}\left(f_{1}-g\right), \tilde{\psi}_{e, m ; j, k}^{\emptyset}\right\rangle\right|+\left|\left\langle f_{1}-g, \tilde{\psi}_{e, m ; j, k-\boldsymbol{\delta}_{1}}^{\{1\}}\right\rangle\right|\right) \\
& \lesssim \inf _{g \in X}\left(2^{-j}\left\|\frac{\partial}{\partial x_{1}}\left(f_{1}-g\right)\right\|_{L_{\infty}\left(\sigma_{j k}\right)}+\left\|\left.\left(f_{1}-g\right)\right|_{x_{1}=2^{-j}\left(k-\boldsymbol{\delta}_{1}\right)}\right\|_{L_{\infty}\left(\mathbb{R}^{n-1}\right)}\right) .
\end{aligned}
$$

Note that $\frac{\partial}{\partial x_{1}} g$ can be chosen independently of $\left.g\right|_{x_{1}=2^{-j}\left(k-\delta_{1}\right)}$. In particular, for $\left.\left(f_{1}-g\right)\right|_{x_{1}=2^{-j}\left(k-\delta_{1}\right)}=0$ and $\frac{\partial}{\partial x_{1}} g \in \Pi_{3}$ one obtains

$$
\left|\left\langle f_{1}, \tilde{\psi}_{e, m ; j, k}^{\{1\}}\right\rangle\right| \lesssim 2^{-j} E_{3}\left(\frac{\partial}{\partial x_{1}} f_{1}, L_{\infty}\left(\sigma_{j k}\right)\right),
$$

which leads again to estimate (3.32). For $\boldsymbol{\alpha}_{e, m}^{\Delta}$ the proof is done analogously. Finally, one shows by similar arguments and Lemma 3.7 that

$$
\left\|\boldsymbol{\beta}_{m, i}\right\|_{\ell_{p}} \lesssim \begin{cases}\left\|f_{i}\right\|_{B_{q}^{s}\left(L_{p}(\mathbb{R})\right),} & \text { if } m_{i}=1, \\ \left\|\frac{\partial}{\partial x_{i}} f_{i}\right\|_{B_{q}^{s}\left(L_{p}(\mathbb{R})\right)}, & \text { if } m_{i}=2 .\end{cases}
$$

Applying biorthogonality we obtain the following result.

Theorem 3.14. Let $\frac{n}{p}<s<\min \left(3,1+\frac{1}{p}\right), 0<p, q \leq \infty$. Then every $\boldsymbol{f} \in$ $\check{\boldsymbol{B}}_{q}^{s}\left(\boldsymbol{L}_{p}\left(\mathbb{R}^{n}\right)\right)$ has the expansion

$$
\boldsymbol{f}=\sum_{m, i, k} \beta_{m, i ; k} \boldsymbol{\varphi}_{m, i ; j_{0}, k}+\sum_{j \geq j_{0}} \sum_{e, m, k}\left(\sum_{i \neq i_{e}} \alpha_{e, m, i ; j, k}^{\nabla} \boldsymbol{\psi}_{e, m, i ; j, k}^{\nabla}+\alpha_{e, m ; j, k}^{\Delta} \boldsymbol{\psi}_{e, m ; j, k}^{\Delta}\right)
$$

with uniquely determined coefficients, and

$$
\|f\|_{\check{\boldsymbol{B}}_{q, p}^{s}} \sim \sum_{m, i}\left\|\boldsymbol{\beta}_{m, i}\right\|_{\ell_{p}}+\sum_{e, m}\left(\left\|\boldsymbol{\alpha}_{e, m}^{\Delta}\right\|_{\ell_{p q}^{s+1}}+\sum_{i \neq i_{e}}\left\|\boldsymbol{\alpha}_{e, m, i}^{\nabla}\right\|_{\ell_{p q}^{s+1}}\right) .
$$

Note that we need the $\ell_{p q}^{s+1}$-norm and not the $\ell_{p q}^{s}$-norm in the statements above. This is due to the normalization of the wavelets in $\check{\boldsymbol{B}}_{q}^{s}\left(\boldsymbol{L}_{p}\left(\mathbb{R}^{n}\right)\right)$.

The limited regularity of the wavelets and their dual functionals permits only a small range of smoothness parameters $s$. In particular, for higher spatial dimensions $n$ there are no admissible values for $s$ if $p$ becomes small. However, in many cases one might only need a one-sided estimate. Therefore, we stated the inequalities in Theorems 3.12 and 3.13 separately.

By the construction of the divergence-free wavelets we know that any linear combination of the wavelets $\boldsymbol{\psi}_{e, m, i ; j, k}^{\nabla}, e \in E^{*}, m \in\{1,2\}^{n}, i \in i_{e}, j, k \in \mathbb{Z}$ is divergence-free (cf. (3.28)). On the other hand, we know from (3.31) that for any divergence-free vector field $\boldsymbol{f}$ the coefficients $\alpha_{e, m ; j, k}^{\Delta}:=\left\langle\boldsymbol{f}, \tilde{\boldsymbol{\psi}}_{e, m ; j, k}^{\Delta}\right\rangle$ are zero. Thus, we know that a vector field $f$ is divergence-free if and only if in the expansion in Theorem 3.14 the coefficients $\alpha_{e, m ; j, k}^{\Delta}$ are zero and the coarse part $\sum_{m, i, k} \beta_{m, i ; k} \boldsymbol{\varphi}_{m, i ; j_{0}, k}$ is divergence-free. For the single-scale wavelet bases we deduce the following result.

Proposition 3.15. The functions

$$
2^{\frac{n j}{2}} \boldsymbol{\psi}_{e, m, i ; j, k}^{\nabla}, \quad i \neq i_{e}, \quad 2^{\frac{n j}{2}} \boldsymbol{\psi}_{e, m ; j, k}^{\Delta}, \quad e \in E_{n}^{*}, m \in\{1,2\}^{n}, k \in \mathbb{Z}^{n}
$$


generate a Riesz basis for $\boldsymbol{W}_{j}$ and $2^{\frac{n j}{2}} \boldsymbol{\psi}_{e, m, i ; 0, k}^{\nabla}, i \neq i_{e}, e \in E_{n}^{*}, m \in\{1,2\}^{n}$, $k \in \mathbb{Z}^{n}$, constitute a Riesz basis for $\boldsymbol{W}_{j}^{\nabla}$, with Riesz bounds independent of $j$.

Proof. It is well known that it is sufficient to show the statement for $j=0$. From the construction in Section 3.3 it is obvious that the functions $\boldsymbol{\psi}_{e, m, i ; 0, k}^{\nabla}$ and $\boldsymbol{\psi}_{e, m ; 0, k}^{\Delta}$ are complete in $W_{0}$. To show stability we consider

$$
\boldsymbol{w}:=\sum_{e, m, k}\left(d_{e, m ; k}^{\Delta} \boldsymbol{\psi}_{e, m ; 0, k}^{\Delta}+\sum_{i \neq i_{e}} d_{e, m, i ; k}^{\nabla} \boldsymbol{\psi}_{e, m, i ; 0, k}^{\nabla}\right) \in W_{0} .
$$

Theorem 3.12 gives

$$
\|\boldsymbol{w}\|_{\boldsymbol{L}_{2}} \lesssim\|\boldsymbol{w}\|_{\check{\boldsymbol{B}}_{2,2}^{s}} \lesssim\|\boldsymbol{d}\|_{\ell_{2}}:=\left(\sum_{e, m, k}\left|d_{e, m ; k}^{\Delta}\right|^{2}+\sum_{i \neq i_{e}}\left|d_{e, m, i ; k}^{\nabla}\right|^{2}\right)^{\frac{1}{2}}
$$

Since $\frac{\partial}{\partial x_{i}} w_{i}$ is continuous we have

$$
\left|d_{e, m, i ; k}^{\nabla}\right|=\left|\left\langle\boldsymbol{w}, \tilde{\boldsymbol{\psi}}_{e, m, i ; 0, k}^{\nabla}\right\rangle\right| \lesssim\left\|\frac{\partial}{\partial x_{i}} w_{i}\right\|_{C(\sigma+k)} \lesssim\left\|w_{i}\right\|_{L_{2}(\sigma+k)},
$$

where supp $\tilde{\boldsymbol{\psi}}_{e, m, i}^{\nabla} \subset \sigma$. Since $\tilde{\boldsymbol{\psi}}_{e, m, i}^{\nabla}$ is compactly supported we conclude that

$$
\sum_{k \in \mathbb{Z}^{n}}\left|d_{e, m, i ; k}^{\nabla}\right|^{2} \lesssim\left\|w_{i}\right\|_{L_{2}(\mathbb{R})}^{2}
$$

With an analogous argument for the coefficients $d_{e, m ; k}^{\Delta}$ we obtain finally that $\|\boldsymbol{d}\|_{\ell_{2}} \lesssim\|\boldsymbol{w}\|_{\boldsymbol{L}_{2}(\mathbb{R})}$, and the first part of the proposition is shown. Furthermore, we know from (3.28) and (3.31) that $\boldsymbol{w}$ is divergence-free if and only if $d_{e, m ; k}^{\Delta}=0$ for all $e \in E_{n}^{*}, m \in\{1,2\}^{n}, k \in \mathbb{Z}^{n}$, which proves the second statement.

3.5. Quasi-interpolation. As already mentioned above, $\Lambda^{ \pm}$interpolates function values and values of derivatives or primitives at the integers. This, however, is not realistic in applications where one is interested in the analysis of experimental data with the aid of wavelets. In fact, experimental data typically consist of measurements of the velocity field at given nodes. This means, that one only has the point values of the velocity field available and no information on the derivatives or primitives. Thus, we study approximation schemes for our divergence-free interpolating Hermite splines. Our goal is to replace $f^{\prime}(k)$ and $\int_{k}^{k+1} f(x) d x$ by linear functionals depending only on function values $f(k), k \in \mathbb{Z}$, in such a way that we still have a good approximation. This condition leads to the notion of quasi-interpolation.

Definition 3.16. Let $S \subset C\left(\mathbb{R}^{n}\right)$. A linear operator $Q: C^{m}\left(\mathbb{R}^{n}\right) \rightarrow S$, is called a quasi-interpolation operator, if:

(a) $Q$ preserves polynomials of degree less than $d \in \mathbb{N}$, i.e., $Q(P)=P$ for $P \in \Pi_{d-1}$.

(b) The operators $Q$ are local and uniformly bounded, i.e., there is a constant $C_{Q}$ and a compact set $K$ so that for each $U \subset \mathbb{R}^{n}$,

$$
\|Q f\|_{L_{p}(U)} \leq C_{Q}\|f\|_{L_{p}(U+K)} .
$$

Theorem 3.17. Let $1 \leq p, q \leq \infty$ and $m+n / p<s \leq d$. The scaled quasiinterpolation operator $Q_{h}$ defined by $Q_{h} f:=Q(f(h \cdot))(\dot{\bar{h}})$, with any $Q$ from Definition 3.16, satisfies for any $f \in B_{q}^{s}\left(L_{p}\left(\mathbb{R}^{n}\right)\right)$ the inequality

$$
\left\|Q_{h} f-f\right\|_{L_{p}(\mathbb{R})} \lesssim h^{s}|f|_{B_{q}^{s}\left(L_{p}\left(\mathbb{R}^{n}\right)\right)} .
$$


Proof. From the assumptions of the theorem we know that $B_{\infty}^{s}\left(L_{p}\left(\mathbb{R}^{n}\right)\right)$ is embedded in $C^{m}\left(\mathbb{R}^{n}\right)$, i.e., $Q_{h} f$ is well defined. With $\sigma_{h, k}=h\left([0,1]^{n}+k\right)$ we have for any $P \in \Pi_{3}$ that

$\left\|Q_{h} f-f\right\|_{L_{p}\left(\sigma_{h, k}\right)}^{p} \leq\left\|Q_{h} f-Q_{h} P\right\|_{L_{p}\left(\sigma_{h, k}\right)}^{p}+\|P-f\|_{L_{p}\left(\sigma_{h, k}\right)}^{p} \leq\left(C_{q}+1\right)\|P-f\|_{L_{p}\left(\sigma_{h, k}^{*}\right)}^{p}$,

where $\sigma_{h, k}^{*}=h\left([0,1]^{n}+k+K\right)$. Hence,

$$
\left\|Q_{h} f-f\right\|_{L_{p}(\mathbb{R})}^{p} \leq \sum_{k \in \mathbb{Z}^{n}}\left(E_{d}\left(f, L_{p}\left(\sigma_{h, k}^{*}\right)\right)\right)^{p} .
$$

Now the statement follows immediately from Lemma 3.6 .

Obviously, $\Lambda_{0}^{+}$and $\Lambda_{0}^{-}$satisfy the conditions of Definition 3.16 with $m=1, d=4$ and $m=0, d=3$, respectively. Now, we set

$$
\begin{aligned}
Q^{+} f(x) & :=\sum_{k \in \mathbb{Z}}\left(f(k) \xi_{1}^{+}(x-k)+q_{k}^{+}(f) \xi_{2}^{+}(x-k)\right), \\
Q^{-} f(x) & :=\sum_{k \in \mathbb{Z} \backslash\{0\}}\left(q_{k}^{-}(f) \xi_{1}^{-}(x-k)+f(k) \xi_{2}^{-}(x-k)\right) .
\end{aligned}
$$

Obviously, $Q^{+}$and $Q^{-}$are quasi-interpolation operators with exactness $d=4$ and $d=3$, respectively, if

$$
q_{k}^{+}(p)=p^{\prime}(k), \quad p \in \Pi_{3}, \quad q_{k}^{-}(p)=\int_{k-1}^{k} p(x) d x, \quad p \in \Pi_{2},
$$

and the functionals $q_{k}^{ \pm}$depend only on values of $f$ in a neighborhood of $k$. Since we only know values of $f$ at the integer points, we have to consider methods for numerical differentiation and integration with a sufficient polynomial exactness in order to define suitable functionals $q_{k}^{+}$and $q_{k}^{-}$, respectively. Local functionals of minimal support, with sufficient polynomial exactness are

$$
\begin{aligned}
& q_{k}^{+}(f)=\frac{1}{6}(-f(k+2)+6 f(k+1)-3 f(k)-2 f(k-1)), \\
& q_{k}^{+}(f)=\frac{1}{6}(f(k-2)-6 f(k-1)+3 f(k)+2 f(k+1)), \\
& q_{k}^{-}(f)=\frac{1}{12}(5 f(k-1)+8 f(k)-f(k+1)), \\
& q_{k}^{-}(f)=\frac{1}{12}(-f(k-2)+8 f(k-1)+5 f(k)) .
\end{aligned}
$$

We also present the corresponding symmetric functionals of minimal support:

$$
\begin{aligned}
& q_{k}^{+}(f)=\frac{1}{12}(f(k-2)-8 f(k-1)+8 f(k+1)-f(k+2)), \\
& q_{k}^{-}(f)=\frac{1}{24}(-f(k-2)+13 f(k-1)+13 f(k)-f(k+1)) .
\end{aligned}
$$

By corresponding scaled versions of $Q^{ \pm}$we are able to obtain an approximation of a smooth function $f$ from sampled data, which has the same order of approximation as the interpolation operators $\Lambda_{j}^{ \pm}$. By tensor product methods we can also construct quasi-interpolation operators replacing the multivariate Hermite interpolation operators $\Lambda_{j}^{I}$ and $\boldsymbol{\Lambda}_{j}$. 


\section{LIFTING SCHEME}

The lifting scheme (yielding so-called second generation wavelets) has become a popular standard tool to adapt wavelet bases to particular requirements, [31] (see also 8 for a quite similar approach called stable completion). The adaptation and usage of second-generation wavelets for the numerical solution of partial differential equations by a collocation method is introduced in 22, 23, 36, 37.

For multiwavelets, the lifting scheme has been applied e.g. in [11, 18. In 2, 19, 20, the lifting scheme has been applied for biorthogonal multiwavelets generated from Hermite splines also yielding wavelets with arbitrary order of vanishing moments.

In this section we show how the lifting scheme can be applied to interpolatory divergence-free wavelets as constructed above. Let $\xi, \eta^{\text {old }}$ be an initial pair of scaling function and corresponding wavelet. Then, the simplest form of a lifting scheme on $\mathbb{R}$ is to define a new wavelet $\eta^{\text {new }}$ by

$$
\eta^{\text {new }}(x):=\eta^{\text {old }}(x)+\sum_{k} u_{k} \xi(x-k),
$$

where $u_{k} \in \mathbb{R}$ are suitably chosen coefficients. The generalization to general interpolation grids and domains is straightforward.

For the Hermite cubic splines the lifting scheme can be described by

$$
\left(\begin{array}{l}
\eta_{1}^{+, \text {new }} \\
\eta_{2}^{+, \text {new }}
\end{array}\right)(x)=\left(\begin{array}{c}
\eta_{1}^{+, \text {old }} \\
\eta_{2}^{+, \text {old }}
\end{array}\right)(x)+\sum_{k} \boldsymbol{U}_{k}^{+}\left(\begin{array}{c}
\xi_{1}^{+} \\
\xi_{2}^{+}
\end{array}\right)(x-k)
$$

with $\boldsymbol{U}_{k}^{+} \in \mathbb{R}^{2 \times 2}$. We leave the choice of these matrices free in order to leave freedom to achieve desirable properties.

Having fixed the wavelets $\eta_{1}^{+, \text {new }}$ and $\eta_{2}^{+, \text {new }}$ we want to choose wavelets $\eta_{1}^{-, \text {new }}$ and $\eta_{2}^{-, \text {new }}$ which permit the construction of divergence-free wavelets. The essential relation for this construction was (3.16). Therefore, we set

$$
\begin{aligned}
\left(\begin{array}{c}
\eta_{1}^{-, \text {new }} \\
\eta_{2}^{-, \text {new }}
\end{array}\right)(x) & =\frac{1}{2} \frac{d}{d x}\left(\begin{array}{c}
\eta_{1}^{+, \text {new }} \\
\eta_{2}^{+, \text {new }}
\end{array}\right) \\
& =\left(\begin{array}{c}
\eta_{1}^{-, \text {old }} \\
\eta_{2}^{-, \text {old }}
\end{array}\right)(x)+\frac{1}{2} \sum_{k} \boldsymbol{U}_{k}^{+}\left(\begin{array}{c}
\xi_{1}^{-}(x-k)-\xi_{1}^{-}(x-k-1) \\
\xi_{2}^{-}(x-k)
\end{array}\right) \\
& =\left(\begin{array}{c}
\eta_{1}^{-, \text {old }} \\
\eta_{2}^{-, \text {old }}
\end{array}\right)(x)+\sum_{k} \boldsymbol{U}_{k}^{-}\left(\begin{array}{c}
\xi_{1}^{-} \\
\xi_{2}^{-}
\end{array}\right)(x-k),
\end{aligned}
$$

where

$$
\boldsymbol{U}_{k}^{-}=\frac{1}{2}\left(\boldsymbol{U}_{k}^{+}-\boldsymbol{U}_{k-1}^{+}\left(\begin{array}{cc}
1 & 0 \\
0 & 0
\end{array}\right)\right)
$$

Since $\eta_{m}^{+, \text {new }}$ and $\eta_{m}^{-, \text {new }}$ satisfy (3.16) the construction of divergence-free wavelets can be done as in Section 3.3 .

That means, we have to fix either the $\boldsymbol{U}_{k}^{+}$or $\boldsymbol{U}_{k}^{-}$in order to establish certain properties of the wavelets $\eta_{m}^{+, \text {new }}$ and $\eta_{m}^{- \text {,new }}$, both. In the particular important case of vanishing moments it turns out that $k$ vanishing moments for $\eta_{m}^{+, \text {new }}$ with 
$\operatorname{supp} \eta_{m}^{+, \text {new }} \subset[-R, R]$ imply

$$
\int_{\mathbb{R}} x^{\ell} \eta_{m}^{-, \text {new }}(x) d x= \begin{cases}-\frac{\ell}{2} \int_{\mathbb{R}} x^{\ell-1} \eta_{m}^{+, \text {new }}(x) d x=0, & \ell=1, \ldots, k, \\ \eta_{m}^{+, \text {new }}(R)-\eta_{m}^{+, \text {new }}(-R)=0, & \ell=0,\end{cases}
$$

i.e., $k+1$ vanishing moments for $\eta_{m}^{-, \text {new }}$.

\section{Appendix A. Proofs from Section 3.2}

In this appendix we collect the proofs of the results from Section 3.2 .

Proof of Lemma 3.4. Obviously, it is sufficient to show the statement for finite right-hand sides. Let us first assume that $1 \leq p<\infty$. For

$$
v_{j}:=\sum_{k \in \mathbb{Z}^{n}} \alpha_{j, k} \phi\left(2^{j} \cdot-k\right)
$$

we have by scaling and the compact support of $\phi$,

$$
\left\|v_{j}\right\|_{L_{p}\left(\mathbb{R}^{n}\right)}=2^{-\frac{j n}{p}}\left\|\sum_{k \in \mathbb{Z}^{n}} \alpha_{j k} \phi(\cdot-k)\right\|_{L_{p}\left(\mathbb{R}^{n}\right)} \lesssim 2^{-\frac{j n}{p}}\left\|\boldsymbol{\alpha}_{j}\right\|_{\ell_{p}}\|\phi\|_{L_{p}\left(\mathbb{R}^{n}\right)} .
$$

Now, (3.24) follows from [9, Thm. 30.2]. Analogously we conclude for $M \in \mathbb{N}$, $M>\sigma$ that

$$
\begin{aligned}
\omega_{M}\left(v_{j}, t, L_{p}\left(\mathbb{R}^{n}\right)\right) & \lesssim 2^{-\frac{j n}{p}}\left\|\boldsymbol{\alpha}_{j}\right\|_{\ell_{p}} \omega_{M}\left(\phi, 2^{j} t, L_{p}\left(\mathbb{R}^{n}\right)\right) \\
& \lesssim 2^{j\left(\sigma-\frac{n}{p}\right)} t^{\sigma}\left\|\boldsymbol{\alpha}_{j}\right\|_{\ell_{p}}\|\phi\|_{B_{\infty}^{\sigma}\left(L_{p}\left(\mathbb{R}^{n}\right)\right)} .
\end{aligned}
$$

Since $\omega_{M}\left(f, t, L_{p}\left(\mathbb{R}^{n}\right)\right) \lesssim\|f\|_{L_{p}\left(\mathbb{R}^{n}\right)}$, we conclude for $0<q<\infty$ that

$$
\begin{aligned}
& \left|\sum_{j=j_{0}}^{\infty} v_{j}\right|_{B_{q}^{s}\left(L_{p}\left(\mathbb{R}^{n}\right)\right)} \lesssim\left(\sum_{r \in \mathbb{Z}}\left(2^{r s} \sum_{j=j_{0}}^{\infty} \omega_{M}\left(v_{j}, 2^{-r}, L_{p}\left(\mathbb{R}^{n}\right)\right)\right)^{q}\right)^{\frac{1}{q}} \\
& \quad \lesssim\left(\sum_{r \in \mathbb{Z}}\left(2^{r s} \sum_{j=r}^{\infty}\left\|v_{j}\right\|_{L_{p}\left(\mathbb{R}^{n}\right)}\right)^{q}\right)^{\frac{1}{q}}+\left(\sum_{r=j_{0}}^{\infty}\left(2^{r(s-\sigma)} \sum_{j=j_{0}}^{r} 2^{j\left(\sigma-\frac{n}{p}\right)}\left\|\boldsymbol{\alpha}_{j}\right\|_{\ell_{p}}\right)^{q}\right)^{\frac{1}{q}} .
\end{aligned}
$$

Furthermore, for any $q \in(0, \infty)$, we have

$$
\left\|\sum_{j=j_{0}}^{\infty} v_{j}\right\|_{L_{p}\left(\mathbb{R}^{n}\right)} \leq \sum_{j=j_{0}}^{\infty} 2^{j s}\left\|v_{j}\right\|_{L_{p}\left(\mathbb{R}^{n}\right)} \leq\left(\sum_{r=j_{0}}^{\infty}\left(2^{r s} \sum_{j=j_{0}}^{r}\left\|v_{j}\right\|_{L_{p}\left(\mathbb{R}^{n}\right)}\right)^{q}\right)^{\frac{1}{q}} .
$$

Thus, substituting $r \rightarrow-r$ and $j \rightarrow-j$ in the second sum we obtain

$$
\begin{aligned}
\left\|\sum_{j=j_{0}}^{\infty} v_{j}\right\|_{B_{q}^{s}\left(L_{p}\left(\mathbb{R}^{n}\right)\right)} \lesssim\left(\sum_{r \in \mathbb{Z}}\right. & \left.\left(2^{r s} \sum_{j=r}^{\infty} 2^{-\frac{j n}{p}}\left\|\boldsymbol{\alpha}_{j}\right\|_{\ell_{p}}\right)^{q}\right)^{\frac{1}{q}} \\
& +\left(\sum_{r \in \mathbb{Z}}\left(2^{r(\sigma-s)} \sum_{j=r}^{\infty} 2^{j\left(\frac{n}{p}-\sigma\right)}\left\|\boldsymbol{\alpha}_{-j}\right\|_{\ell_{p}}\right)^{q}\right)^{\frac{1}{q}} .
\end{aligned}
$$

Now, (3.25) follows from the discrete Hardy inequality [15, Chap. 2, Lemma 3.4].

For $p=\infty$ or $q=\infty$ the proof is analogous, and for $p<1$ the use of $\|x+y\|_{p}^{p} \leq$ $\|x\|_{\ell_{p}}^{p}+\|y\|_{p}^{p}$ instead of Minkowski's inequality shows the assertion. 
Proof of Lemma 3.6. If $\tau<p$, we can apply Hölder's inequality to obtain

$$
2^{j\left(\frac{n}{\tau}-\frac{n}{p}\right)}\left\|f^{(\alpha)}\right\|_{L_{\tau}\left(\sigma_{j k}\right)} \leq\left\|f^{(\alpha)}\right\|_{L_{p}\left(\sigma_{j k}\right)}, \quad|\alpha| \leq \mu,
$$

i.e., it is sufficient to prove the claim for $p \leq \tau$. For $P \in \Pi_{d-1}$ and $T_{j k} f(x)=$ $f\left(2^{-j}(x+k)\right)$ we have by $B_{1}^{\mu}\left(L_{\tau}(\sigma)\right) \subset W_{\tau}^{\mu}(\sigma)$ (e.g., [4, Sect. 6.2]), that

$$
\begin{aligned}
\|f-P\|_{W_{\tau}^{\mu}\left(\sigma_{j k}\right)} & =2^{j\left(\mu-\frac{n}{\tau}\right)}\left\|T_{j k}(f-P)\right\|_{W_{\tau}^{\mu}(\sigma)} \leq C 2^{j\left(\mu-\frac{n}{\tau}\right)}\left\|T_{j k}(f-P)\right\|_{B_{1}^{\mu}\left(L_{\tau}(\sigma)\right)} \\
& \leq C 2^{j \mu}\left(\|f-P\|_{L_{\tau}\left(\sigma_{j k}\right)}+2^{-\frac{j n}{\tau}} \sum_{r=0}^{\infty} 2^{r \mu} \omega_{d}\left(T_{j k} f, 2^{-r}, L_{\tau}(\sigma)\right)\right),
\end{aligned}
$$

with a constant $C$ independent of $j$ and $k$. Let the side length of the cube $\sigma$ be $2^{\nu}$. Applying the Whitney-type inequality $E_{d}\left(f, L_{\tau}\left(\sigma_{j k}\right)\right) \lesssim \omega_{d}\left(f, 2^{\nu-j}, L_{\tau}\left(\sigma_{j k}\right)\right.$ ) (see e.g. [9, (25.24)]) and the identity $\omega_{d}\left(T_{j k} f, 2^{-r}, L_{\tau}(\sigma)\right)=2^{\frac{j n}{\tau}} \omega_{d}\left(f, 2^{-j-r}, L_{\tau}\left(\sigma_{j k}\right)\right)$, we obtain

$$
E_{d}\left(f, W_{\tau}^{\mu}\left(\sigma_{j k}\right)\right) \lesssim \sum_{r=j}^{\infty} 2^{r \mu} \omega_{d}\left(f, 2^{\nu-r}, L_{\tau}\left(\sigma_{j k}\right)\right) .
$$

For $\mu=0$ the estimate follows immediately from the Whitney-type estimate.

From [9, Theorem 30.5] we conclude

$$
\begin{aligned}
E_{d}\left(f, W_{\tau}^{\mu}\left(\sigma_{j k}\right)\right) & \lesssim \sum_{r=j}^{\infty} 2^{r \mu} \sum_{\ell=r-\nu}^{\infty} 2^{n \ell\left(\frac{1}{p}-\frac{1}{\tau}\right)} \omega_{d}\left(f, 2^{-\ell}, L_{p}\left(\sigma_{j k}\right)\right) \\
& =\sum_{\ell=j-\nu}^{\infty} \underbrace{\sum_{r=j}^{\ell+\nu} 2^{r \mu}}_{\lesssim 2^{\ell \mu}} 2^{n \ell\left(\frac{1}{p}-\frac{1}{\tau}\right)} \omega_{d}\left(f, 2^{-\ell}, L_{p}\left(\sigma_{j k}\right)\right) .
\end{aligned}
$$

Together with (A.1) for $\tau=p$ we have

$$
E_{d}\left(f, W_{\tau}^{\mu}\left(\sigma_{j k}\right)\right) \lesssim \sum_{r=j-\nu}^{\infty} 2^{r\left(\mu+\frac{n}{p}-\frac{n}{\tau}\right)} \omega_{d}\left(f, 2^{-r}, L_{p}\left(\sigma_{j k}\right)\right) .
$$

For $1 \leq p<\infty$ we conclude by Minkowski's inequality and [9, (26.14)] that

$$
\begin{aligned}
\left(\sum_{k \in \mathbb{Z}^{n}} E_{d}\left(f, W_{\tau}^{\mu}\left(\sigma_{j k}\right)\right)^{p}\right)^{\frac{1}{p}} & \lesssim \sum_{r=j-\nu}^{\infty} 2^{r\left(\mu+\frac{n}{p}-\frac{n}{\tau}\right)}\left(\sum_{k \in \mathbb{Z}} \omega_{d}\left(f, 2^{-r}, L_{p}\left(\sigma_{j k}\right)\right)^{p}\right)^{\frac{1}{p}} \\
& \lesssim \sum_{r=j-\nu}^{\infty} 2^{r\left(\mu+\frac{n}{p}-\frac{n}{\tau}\right)} \omega_{d}\left(f, 2^{-r}, L_{p}(\mathbb{R})\right)=: b_{j-\nu} .
\end{aligned}
$$

Now, by the discrete Hardy inequality [15, Chap. 2, Lemma 3.4] we obtain (for $0<q<\infty)$

$$
\begin{aligned}
\sum_{j \in \mathbb{Z}}\left(2^{j\left(s-\frac{n}{p}+\frac{n}{\tau}-\mu\right)}\left(\sum_{k \in \mathbb{Z}^{n}} E_{d}\left(f, W_{\tau}^{\mu}\left(\sigma_{j k}\right)\right)^{p}\right)^{\frac{1}{p}}\right)^{q} \lesssim \sum_{j \in \mathbb{Z}}\left(2^{j\left(s-\frac{n}{p}+\frac{n}{\tau}-\mu\right)} b_{j}\right)^{q} \\
\lesssim \sum_{j \in \mathbb{Z}}\left(2^{j s} \omega_{d}\left(f, 2^{-j}, L_{p}(\mathbb{R})\right)\right)^{q} \lesssim|f|_{B_{q}^{s}\left(L_{p}(\mathbb{R})\right)} .
\end{aligned}
$$

For $p=\infty$ or $q=\infty$ the estimate works analogously. For $p<1$ Minkowski's inequality is replaced by $\|x+y\|_{p}^{p} \leq\|x\|_{\ell_{p}}^{p}+\|y\|_{p}^{p}$ and the remainder of the proof follows the above lines. 
Proof of Lemma 3.7. By Hölders inequality we conclude that it is sufficient to prove the result for $p \leq \tau$. For $s>\alpha>\frac{1}{p}-\frac{1}{\tau}+\mu$ we know that $B_{q}^{\alpha}\left(L_{p}\left(\sigma_{k}\right)\right)$ is embedded in the Sobolev space $W_{\tau}^{\mu}\left(\sigma_{k}\right)$, i.e.,

$$
\|f\|_{W_{\tau}^{\mu}\left(\sigma_{k}\right)}^{p} \lesssim\|f\|_{L_{p}\left(\sigma_{k}\right)}+|f|_{B_{q}^{\alpha}\left(L_{p}\left(\sigma_{k}\right)\right)} .
$$

Now the statement follows as in the proof of Lemma 3.6 .

Proof of Corollary 3.8. Obviously $\left|\left\langle f, \tilde{\xi}_{m ; j_{0}, k}^{+}\right\rangle\right| \lesssim\left\|f^{(m-1)}\right\|_{L_{\infty}\left(2^{\left.-j_{0}[k, k+1]\right)}\right.}$ and since $\Pi_{3} \subset V_{j}^{+}$we know $\tilde{\eta}_{m ; j, k}^{+}(P)=0, P \in \Pi_{3}$. Thus,

$$
\begin{aligned}
\left|\left(f, \tilde{\eta}_{m ; j, k}^{+}\right)\right| & =\inf _{P \in \Pi_{3}}\left|\left\langle f-P, \tilde{\eta}_{i ; j, k}^{+}\right\rangle\right| \lesssim E_{4}\left(f, L_{\infty}\left(\sigma_{j k}\right)\right)+2^{-j} E_{3}\left(f^{\prime}, L_{\infty}\left(\sigma_{j k}\right)\right) \\
& \lesssim E_{4}\left(f, W_{\infty}^{1}\left(\sigma_{j k}\right)\right),
\end{aligned}
$$

with $\sigma_{j k}=2^{-j}[k, k+1]$. Now, the statement follows immediately from Lemma 3.6 and Lemma 3.7 .

For $\tilde{\xi}_{m ; j_{0}, k}^{-}$and $\tilde{\eta}_{m ; j, k}^{-}$we obtain, analogously,

$$
\left|\left\langle f, \tilde{\xi}_{1 ; j_{0}, k}^{-}\right\rangle\right| \lesssim\|f\|_{L_{1}\left(2^{\left.-j_{0}[k-1, k]\right)}\right.} \quad \text { and } \quad\left|\left\langle f, \tilde{\xi}_{2 ; j_{0}, k}^{-}\right\rangle\right| \lesssim\|f\|_{L_{\infty}\left(2^{\left.-j_{0}[k-1, k]\right)}\right.}
$$

as well as

$$
\left|\left\langle f, \tilde{\eta}_{m ; j, k}^{-}\right\rangle\right| \lesssim E_{3}\left(f, L_{\infty}\left(\sigma_{j k}\right)\right)+2^{j} E_{3}\left(f, L_{1}\left(\sigma_{j k}\right)\right)
$$

Next, Hölder's inequality yields $\|f-P\|_{L_{1}\left(\sigma_{j k}\right)} \lesssim 2^{-j}\|f-P\|_{L_{\infty}\left(\sigma_{j k}\right)}$, i.e.,

$$
\left|\left\langle f, \tilde{\eta}_{m ; j, k}^{-}\right\rangle\right| \lesssim E_{3}\left(f, L_{\infty}\left(\sigma_{j k}\right)\right) \text {. }
$$

Now the statement follows as before from Lemma 3.6 and Lemma 3.7

\section{REFERENCES}

1. C.M. Albukrek, K. Urban, D. Rempfer, and J.L. Lumley, Divergence-free wavelet analysis of turbulent flows, J. Sci. Comput. 17 (2002), no. 1-4, 49-66. MR1910551

2. A.Z. Averbuch and V.A. Zheludev, Lifting scheme for biorthogonal multiwavelets originated from Hermite splines, IEEE Trans. Signal Process. 50 (2002), no. 3, 487-500. MR.1895057 (2003b:94004)

3. G. Battle and P. Federbush, Divergence-free vector wavelets, Michigan Math. J. 40 (1993), no. 1, 181-195. MR1214063 (94c:42021)

4. J. Bergh and J. Löfström, Interpolation spaces: An introduction, Springer, Berlin, 1976. MR.0482275 (58:2349)

5. O. V. Besov, V. P. Il'in, and S. M. Nikol'skiř, Integral representation of functions and imbedding theorems, vol. II, Winston \& Sons, Washington, D.C., 1979. MR0521808 (80f:46030b)

6. Claudio Canuto, Anita Tabacco, and Karsten Urban, The wavelet element method. I. Construction and analysis, Appl. Comput. Harmon. Anal. 6 (1999), no. 1, 1-52. MR1664902 (99k:42055)

7. , The wavelet element method. II. Realization and additional features in $2 D$ and 3D, Appl. Comput. Harmon. Anal. 8 (2000), no. 2, 123-165. MR.1743533(2001e:42044)

8. J. M. Carnicer, W. Dahmen, and J. M. Peña, Local decomposition of refinable spaces and wavelets, Appl. Comput. Harmon. Anal. 3 (1996), no. 2, 127-153. MR.1385049 (97f:42050)

9. A. Cohen, Wavelet methods in numerical analysis, Handbook of numerical analysis, Vol. VII, Handbook Numer. Anal., VII, North-Holland, Amsterdam, 2000, pp. 417-711. MR 1804747 (2002c:65252)

10. A. Cohen and R. Masson, Wavelet adaptive method for second order elliptic problems: boundary conditions and domain decomposition, Numer. Math. 86 (2000), no. 2, 193-238. MR.1777487 (2001j:65185)

11. W. Dahmen, B. Han, R.-Q. Jia, and A. Kunoth, Biorthogonal multiwavelets on the interval: Cubic hermite splines, Constr. Approx. 16 (2000), no. 2, 221-259. MR.1735242(2001a:42036) 
12. Wolfgang Dahmen and Reinhold Schneider, Composite wavelet bases for operator equations, Math. Comp. 68 (1999), no. 228, 1533-1567. MR.1648379 (99m:65122)

13. Wavelets on manifolds. I. Construction and domain decomposition, SIAM J. Math. Anal. 31 (1999), no. 1, 184-230 (electronic). MR.1742299 (2000k:65242)

14. Wolfgang Dahmen and Rob Stevenson, Element-by-element construction of wavelets satisfying stability and moment conditions, SIAM J. Numer. Anal. 37 (1999), no. 1, 319-352 (electronic). MR.1742747 (2001c:65144)

15. R. A. DeVore and G. G. Lorentz, Constructive approximation, Springer, New York, 1993. MR.1261635 (95f:41001)

16. D. Donoho, Interpolating wavelet transforms, Preprint, Standford University, 1992.

17. P. Federbush, Navier and Stokes meet the wavelet, Comm. Math. Phys. 155 (1993), no. 2, 219-248. MR1230026 (94g:35171)

18. S.S. Goh, Q. Jiang, and T. Xia, Construction of biorthogonal multiwavelets using the lifting scheme, Appl. Comput. Harmon. Anal. 9 (2000), no. 3, 336-352. MR1793422 (2001h:42052)

19. B. Han, Hermite interpolants and biorthogonal multiwavelets with arbitrary order of vanishing moments, Wavelet Applications in Signal and Image Processing VII (A. Aldroubi, M.A. Unser, and A.F. Laine, eds.), vol. 3813, Proc. SPIE, 1999, pp. 147-161.

20. B. Han, T.P.-Y. Yu, and B. Piper, Multivariate refinable Hermite interpolant, Math. Comp. 73 (2004), no. 248, 1913-1935 (electronic). MR2059743(2005e:41004)

21. C. Heil, G. Strang, and V. Strela, Approximation of translates of refinable functions, Numer. Math. 73 (1996), 75-94. MR1379281 (97c:65033)

22. N.K.-R. Kevlahan and O.V. Vasilyev, An adaptive wavelet collocation method for fluidstructure interaction at high reynolds numbers, SIAM J. Scient. Comput. 26 (2005), 18941915. MR.2196581 (2006h:76072)

23. F. Koster, M. Griebel, N. K.-R. Kevlahan, M. Farge, and K. Schneider, Towards an adaptive wavelet-based 3D Navier-Stokes solver, Numerical flow simulation, I (Marseille, 1997), Notes Numer. Fluid Mech., vol. 66, Vieweg, Braunschweig, 1998, pp. 339-364. MR1668783 (99i:76123)

24. J.D. Lakey, P.R. Massopust, and M.C. Pereyra, Divergence-free multiwavelets, Approximation theory IX, Vol. 2 (Nashville, TN, 1998), Innov. Appl. Math., Vanderbilt Univ. Press, Nashville, TN, 1998, pp. 161-168. MR.1744404

25. J.D. Lakey and M.C. Pereyra, Divergence-free multiwavelets on rectangular domains, Wavelet analysis and multiresolution methods (Urbana-Champaign, IL, 1999), Lecture Notes in Pure and Appl. Math., vol. 212, Dekker, New York, 2000, pp. 203-240. MR1777994 (2001h:42055)

26. P.G. Lemarié-Rieusset, Analyses multi-résolutions non orthogonales, commutation entre projecteurs et dérivation et ondelettes vecteurs à divergence nulle, Rev. Mat. Iberoamericana 8 (1992), no. 2, 221-237. MR1191345 (94d:42044)

27. _ Un théorème d'inexistence pour les ondelettes vecteurs à divergence nulle, $\mathrm{C} . \mathrm{R}$. Acad. Sci. Paris Sér. I Math. 319 (1994), no. 8, 811-813. MR.1300948 (95h:42028)

28. _ Recent developments in the Navier-Stokes problem, Chapman \& Hall/CRC Research Notes in Mathematics, vol. 431, Chapman \& Hall/CRC, Boca Raton, FL, 2002. MR1938147 (2004e:35178)

29. C.A. Micchelli, Interpolatory subdivision schemes and wavelets, J. Approx. Theory 86 (1996), 41-71. MR 1397613 (97g:42030)

30. L. L. Schumaker, Spline functions: Basic theory, Wiley-Interscience, New York, 1981. MR $606200(82 \mathrm{j}: 41001)$

31. W. Sweldens, The lifting scheme: A construction of second generation wavelets, SIAM J. Math. Anal. 29 (1998), no. 2, 511-546 (electronic). MR.1616507 (99e:42052)

32. K. Urban, On divergence-free wavelets, Adv. Comput. Math. 4 (1995), no. 1-2, 51-81. MR.1338895 (96e:42035)

33. Wavelet bases in $H$ (div) and $H$ (curl), Math. Comp. 70 (2001), no. 234, 739-766. MR 1710628 (2001g:42069)

34. W Wavelets in numerical simulation, Lecture Notes in Computational Science and Engineering, vol. 22, Springer-Verlag, Berlin, 2002, Problem adapted construction and applications. MR $1918770(2003 \mathrm{e}: 42001)$

35. O. V. Vasilyev and N. K.-R. Kevlahan, Hybrid wavelet collocation - Brinkman penalization method for complex geometry flows, Internat. J. Numer. Methods Fluids 40 (2002), no. 3-4, 
531-538, ICFD Conference on Numerical Methods for Fluid Dynamics, Part II (Oxford, 2001). MR.1932995

36. O.V. Vasilyev and C. Bowman, Second-generation wavelet collocation method for the solution of partial differential equations, J. Comput. Phys. 165 (2000), no. 2, 660-693. MR.1807301 (2002j:65119)

37. O.V. Vasilyev and N.K.-R. Kevlahan, An adaptive multilevel wavelet collocation method for elliptic problems, J. Comput. Phys. 206 (2005), 412-431. MR2143325(2006a:65173)

University of Ulm, Institute for Numerical Mathematics, Helmholtzstr. 18, D-89069 Ulm, Germany

E-mail address: kai.bittner@uni-ulm.de

University of Ulm, Institute for Numerical Mathematics, Helmholtzstr. 18, D-89069 Ulm, Germany

E-mail address: karsten.urban@uni-ulm.de 